CAB-IB/2900206, IFT-UAM/CSIC-06-05

\title{
More Dual Fluxes and Moduli Fixing
}

\author{
G. Aldazabal ${ }^{a}$, P.G. Cámara ${ }^{b}$, A. Font ${ }^{b 1}$ and L.E. Ibáñez ${ }^{b}$ \\ ${ }^{a}$ Instituto Balseiro, CNEA, Centro Atómico Bariloche, \\ 8400 S.C. de Bariloche, and CONICET, Argentina. \\ ${ }^{b}$ Departamento de Física Teórica C-XI and Instituto de Física Teórica C-XVI, \\ Universidad Autónoma de Madrid, Cantoblanco, 28049 Madrid, Spain
}

\begin{abstract}
We generalize the recent proposal that invariance under T-duality leads to additional nongeometric fluxes required so that superpotentials in type IIA and type IIB orientifolds match. We show that invariance under type IIB S-duality requires the introduction of a new set of fluxes leading to further superpotential terms. We find new classes of $\mathcal{N}=1$ supersymmetric Minkowski vacua based on type IIB toroidal orientifolds in which not only dilaton and complex moduli but also Kähler moduli are fixed. The chains of dualities relating type II orientifolds to heterotic and M-theory compactifications suggests the existence of yet further flux degrees of freedom. Restricting to a particular type IIA/IIB or heterotic compactification only some of these degrees of freedom have a simple perturbative and/or geometric interpretation.
\end{abstract}

\footnotetext{
${ }^{1}$ On leave from Departamento de Física, Facultad de Ciencias, Universidad Central de Venezuela, A.P. 20513, Caracas 1020-A, Venezuela.
} 


\section{Introduction}

Fluxes of antisymmetric fields in string compactifications have been studied intensively in the last few years [1]. One of the most interesting aspects of the presence of these fluxes is that they may generate superpotential couplings for the compactification moduli [2]. These superpotentials, perhaps supplemented by other dynamical effects like gaugino condensation, may lead to a full determination of all moduli, solving an outstanding problem in string theory.

Type IIA orientifolds with fluxes were rather neglected in the past but are are starting to receive more attention $[3,4,5,6,7,8,9,10]$. In IIA compactifications it is possible to switch on backgrounds of even RR and odd NS forms. This in turn implies the important result that IIA flux-induced superpotentials depend on all geometrical moduli as well as on the dilaton. In particular, it has been shown that in simple toroidal orientifolds one can stabilize all closed string moduli in AdS space without considering extra nonperturbative (e.g. gaugino condensation) effects $[5,7,8]$. Moreover, in type IIA it is natural to incorporate metric fluxes $[5,6]$ that correspond to generalized Scherk-Schwarz reductions $[11,12,13,14,15]$. In presence of metric backgrounds, the flux contribution to $R R$ tadpoles can have either sign or even vanish, opening interesting possibilities for model-building [8].

A logical question is whether type IIB flux-induced superpotentials can also depend on all moduli. In fact, it has been recently shown [16] that in order to recover T-duality invariance between the type IIA and type IIB versions of the same compactification in the presence of RR, NS and metric backgrounds, new 'non-geometric' fluxes have to be introduced. Once this is done the superpotentials on the type IIA and type IIB sides adequately match. Such non-geometric fluxes had already been, and continue to be, studied by several authors $[17,18,19,20,21,22,23,15,24,25,26]$. In this paper we generalize the work of [16] to orientifolds with several diagonal geometrical moduli. We will give explicit expressions for the superpotential and tadpoles in terms of integrals involving the flux tensors.

As pointed out in [16], there is still a puzzle. We know that the type IIB theory has an 
S-duality symmetry built in. This symmetry is inherited by the effective potential of type IIB orientifold compactifications in the presence of standard RR and NS backgrounds. However the symmetry disappears if we introduce the new non-geometric fluxes. In the present paper we argue that in order to recover S-duality in the underlying orientifold theory one has to introduce an extra set of 'S-dual' fluxes. These S-dual fluxes give rise to new terms in the effective superpotential and do also contribute to RR tadpoles and Bianchi conditions. In order to study the structure of tadpoles and Bianchi identities we make use of $S L(2, \mathbb{Z})_{S}$ transformations. Whereas the '3-brane' RR tadpole is S-duality invariant, '7-brane' tadpoles come in a $S L(2, \mathbb{Z})_{S}$ triplet and couple to a triplet of 8-forms in agreement with results in $[27,28,29,30]$. On the other hand, S-duality transformations on Bianchi identities give rise to new constraints. The extra flux degrees of freedom still respect T-duality among type IIA and type IIB so that the effective action is both Sduality and T-duality invariant. We describe all these fluxes in the context of a simple $\mathrm{T}^{6} /\left(\Omega(-1)^{F_{L}} I_{6}\right)$ type IIB orientifold $\left(\mathrm{T}^{6} /\left(\Omega(-1)^{F_{L}} I_{3}\right)\right.$ in type IIA) and concentrate on the dynamics of the seven diagonal moduli. The additional S-dual terms in the superpotential allow us to obtain new classes of $\mathcal{N}=1$ Minkowski vacua in which not only dilaton and complex moduli but also Kähler moduli are fixed. As it happened in the AdS type IIA vacua in ref.[8], the contribution of fluxes to RR tadpoles may have the same or opposite sign to that of D-branes.

We also argue that comparison with related compactifications leading to similar models (based on M-theory compactified on $X_{7}=\mathrm{T}^{7} / \mathbb{Z}_{2} \times \mathbb{Z}_{2} \times \mathbb{Z}_{2}$ and heterotic on twisted tori) points at the existence of further flux degrees of freedom beyond those mentioned above. In particular, the combination of type I-heterotic duality with heterotic self T-duality suggests that the full underlying duality symmetry in these toroidal examples includes $S L(2, \mathbb{Z})^{7}=S L(2, \mathbb{Z})_{S} \times S L(2, \mathbb{Z})_{U}^{3} \times S L(2, \mathbb{Z})_{T}^{3}$. Full duality invariance requires the presence of up to $2^{7}$ fluxes.

The structure of this paper is as follows. In the next chapter we define the geometry of our orientifold examples and describe the fluxes, superpotential, RR tadpoles and Bianchi conditions for both the type IIA and its T-dual type IIB version. In chapter 3 we generalize the setting and describe in detail how T-duality requires the introduction of non-geometric 
fluxes and give explicit formulae for the superpotential in this generalized case. The modifications of RR tadpole conditions as well as Bianchi identities are discussed for the three T-dual settings of type IIB with O3-planes, type IIA with O6-planes, and type IIB with O9-planes. We also briefly describe some general properties of minima of the scalar potential induced by fluxes. In chapter 4 we describe how the S-duality underlying type IIB theory requires the introduction of novel 'S-dual' flux degrees of freedom giving rise to new terms in the superpotential. In order to study the structure of tadpoles and Bianchi identities we make use of $S L(2, \mathbb{Z})_{S}$ transformations and provide particular solutions of the constraints. We report on chapter 5 on a search for $\mathcal{N}=1$ Minkowski minima of the flux-induced scalar potential showing several examples. In chapter 6 we compare the type II results with those coming from compactifying M-theory and heterotic strings on twisted tori. We also discuss the generalization to $S L(2, \mathbb{Z})^{7}$ invariant superpotentials in which fluxes fill an spinorial representation as described in the appendix. Some final comments are left for chapter 7 .

\section{Orientifolds with NS, RR and geometric fluxes}

Before addressing the issue of non-geometric fluxes as well as the new fluxes implied by S-duality, we review in this chapter the basic features of the toroidal orientifolds under consideration. We start by describing the moduli and fluxes of a type IIA orientifold on $\mathrm{T}^{6} /\left[\Omega_{P}(-1)^{F_{L}} \sigma_{A}\right]$. In this case the orientifold symmetry allows to include metric fluxes. We then move to the T-dual IIB orientifold on $\mathrm{T}^{6} /\left[\Omega_{P}(-1)^{F_{L}} \sigma_{B}\right]$. For both cases we give the expressions for the flux-induced moduli superpotential as well as Bianchi and RR tadpole cancellation conditions.

\subsection{Notation}

Let us first fix our notation for the geometric moduli on the tori. We focus on compactifications on a factorized torus $\mathrm{T}^{6}=\otimes_{i=1}^{3} \mathrm{~T}_{i}^{2}$. As basis of closed 3-forms with one leg on each sub-torus we take

$$
\alpha_{0}=d x^{1} \wedge d x^{2} \wedge d x^{3} \quad ; \quad \beta_{0}=d y^{1} \wedge d y^{2} \wedge d y^{3}
$$




$$
\begin{aligned}
& \alpha_{1}=d x^{1} \wedge d y^{2} \wedge d y^{3} ; \quad \beta_{1}=d y^{1} \wedge d x^{2} \wedge d x^{3} \\
& \alpha_{2}=d y^{1} \wedge d x^{2} \wedge d y^{3} ;
\end{aligned}
$$

where $y^{i}=x^{i+3}$. Our normalization is $\int_{\mathrm{T}^{6}} \alpha_{I} \wedge \beta_{J}=\delta_{I J}$. The closed 2-forms and their dual 4-forms are

$$
\omega_{i}=-d x^{i} \wedge d y^{i} \quad ; \quad \widetilde{\omega}_{i}=d x^{j} \wedge d y^{j} \wedge d x^{k} \wedge d y^{k} \quad ; i \neq j \neq k
$$

Notice that $\int_{\mathrm{T}^{6}} \omega_{i} \wedge \widetilde{\omega}_{j}=\delta_{i j}$. Each sub-torus $\mathrm{T}_{j}^{2}$ has area $(2 \pi)^{2} A_{j}$ and the geometric complex structure parameters are given by

$$
\tau_{j}=\frac{1}{e_{j x}^{2}}\left(A_{j}+i e_{j x} \cdot e_{j y}\right)
$$

where $e_{j x}$ and $e_{j y}$ are the lattice vectors of sizes $R_{x}^{j}$ and $R_{y}^{j}$. The Kähler form is

$$
J=\sum_{i=1}^{3} A_{i} \omega_{i}
$$

As usual the holomorphic 3-form can be written as

$$
\Omega=\left(d x^{1}+i \tau_{1} d y^{1}\right) \wedge\left(d x^{2}+i \tau_{2} d y^{2}\right) \wedge\left(d x^{3}+i \tau_{3} d y^{3}\right)
$$

Clearly, $\Omega$ can be expanded in the basis of 3 -forms.

\subsection{IIA orientifold with O6-planes}

Consider the IIA orientifold on $\mathrm{T}^{6} /\left[\Omega_{P}(-1)^{F_{L}} \sigma_{A}\right]$, where $\Omega_{P}$ is the world-sheet parity operator and $(-1)^{F_{L}}$ is the space-time fermionic number for left-movers. The involution $\sigma_{A}$ acts on the Kähler form and the holomorphic 3-form as $\sigma_{A}(J)=-J$ and $\sigma_{A}(\Omega)=\Omega^{*}$. In terms of the coordinates this is $\sigma_{A}\left(x^{i}\right)=x^{i}$ and $\sigma_{A}\left(y^{i}\right)=-y^{i}$. These imply O6-planes that span space-time and the $x^{i}$ directions. Each sub-torus has now a square lattice, consistent with the involution. Thus, $\tau_{j}=R_{y}^{j} / R_{x}^{j}$ and $A_{j}=R_{x}^{j} R_{y}^{j}$.

We concentrate our analysis on the seven diagonal moduli of this IIA orientifold, the dilaton $S$, three Kähler moduli $T_{i}$ and three complex structure moduli $U_{i}$. As shown in 
[4] they can be concisely described in terms of the complexified forms

$$
\begin{aligned}
J_{c} & =B+i J=i \sum_{i=1}^{3} T_{i} \omega_{i} \\
\Omega_{c} & =C_{3}+i \operatorname{Re}(C \Omega)=i S \alpha_{0}-i \sum_{i=1}^{3} U_{i} \alpha_{i} .
\end{aligned}
$$

Here $B$ is the NS 2-form whereas $C_{3}$ is the RR 3-form that is even under $\sigma_{A}$ and can therefore be expanded in the $\alpha_{J}$. The compensator field $C$ is specified by

$$
C=e^{-\phi_{4}} e^{K_{c s} / 2} \quad ; \quad K_{c s}=-\log \left[-\frac{i}{8} \int_{\mathrm{T}^{6}} \Omega \wedge \Omega^{*}\right]
$$

where $\phi_{4}$ is the T-duality invariant four-dimensional dilaton given by $e^{\phi_{4}}=e^{\phi} / \sqrt{\operatorname{vol~T}^{6}}$. Clearly, $\operatorname{Re} T_{j}=R_{x}^{j} R_{y}^{j}$ and from $\Omega_{c}$ we readily find

$$
\operatorname{Re} S=e^{-\phi} R_{y}^{1} R_{y}^{2} R_{y}^{3} \quad ; \quad \operatorname{Re} U_{i}=e^{-\phi} R_{x}^{i} R_{y}^{j} R_{y}^{k} \quad ; \quad i \neq j \neq k
$$

We are measuring all lengths in units of $\sqrt{\alpha^{\prime}}$.

The Kähler potential for the moduli takes the usual form

$$
K=-\log \left(S+S^{*}\right)-\sum_{i=1}^{3} \log \left(U_{i}+U_{i}^{*}\right)-\sum_{i=1}^{3} \log \left(T_{i}+T_{i}^{*}\right)
$$

A superpotential is generated by turning on fluxes as we discuss next.

Under the orientifold involution the NS $H_{3}$ is odd, the RR forms $F_{0}$ and $F_{4}$ are even while $F_{2}$ and $F_{6}$ are odd. Thus the general fluxes allowed are

$$
\begin{aligned}
& \bar{H}_{3}=\sum_{L=0}^{3} h_{L} \beta_{L} ; \\
& \bar{F}_{0}=-m ; \quad \bar{F}_{2}=\sum_{i=1}^{3} q_{i} \omega_{i} ; \bar{F}_{4}=\sum_{i=1}^{4} e_{i} \widetilde{\omega}_{i} ; \quad \bar{F}_{6}=e_{0} \alpha_{0} \wedge \beta_{0} .
\end{aligned}
$$

The coefficients in these expansions are integers since the integrals of the fluxes over the corresponding $p$-cycles are quantized. To avoid subtleties with exotic orientifold planes we take the flux integers to be even. As in [8] we take all forms to have dimensions (length) ${ }^{-1}$ so that moduli fields are all dimensionless.

The orientifold involution also allows for metric fluxes that are deformations of the original manifold. Such backgrounds appear naturally in the context of Scherk-Schwarz 
reductions [11]. They are equivalent $[12,13,14,15]$ to compactification on a twisted torus defined by

$$
d \eta^{P}=-\frac{1}{2} \omega_{M N}^{P} \eta^{M} \wedge \eta^{N} \quad ; \quad M, N, P=1, \cdots, 6
$$

where $\eta^{P}$ are the tangent 1-forms. The metric fluxes are the constant coefficients $\omega_{M N}^{P}$, antisymmetric in the lower indices. In general, the twisted torus has isometries with generators $Z_{M}$. The $\omega_{M N}^{P}$ turn out to be the structure constants of the Lie algebra generated by the $Z_{M}$, i.e.

$$
\left[Z_{M}, Z_{N}\right]=\omega_{M N}^{P} Z_{P}
$$

Either from the Jacobi identity of the algebra or from the Bianchi identity of (2.12) one finds that the metric fluxes must satisfy

$$
\omega_{[M N}^{P} \omega_{R] P}^{S}=0
$$

It can further be shown that $\omega_{P N}^{P}=0$ [11]. The metric fluxes must be quantized by consistency of the twisted torus structure [31].

An useful result in the following is that we can contract the metric fluxes with a $p$-form $\mathcal{X}$ to obtain a $(p+1)$-form $\omega \mathcal{X}$ with components

$$
(\omega \mathcal{X})_{L M N_{1} \cdots N_{p-1}}=\omega_{[L M}^{A} \mathcal{X}_{\left.N_{1} \cdots N_{p-1}\right] A}
$$

Actually, for a constant form, $\omega \mathcal{X}$ is basically $d \mathcal{X}$ computed in the twisted torus.

The metric fluxes are even under the orientifold involution. Then they can be of type $\omega_{a b}^{i}, \omega_{j k}^{i}, \omega_{i b}^{a}, i=1,2,3, a=4,5,6$. As in the case of RR and NS fluxes, we only switch on metric fluxes with one leg on each sub-torus. Thus, there are twelve free parameters for which we use the notation

$$
\left(\begin{array}{c}
\omega_{56}^{1} \\
\omega_{64}^{2} \\
\omega_{45}^{3}
\end{array}\right)=\left(\begin{array}{l}
a_{1} \\
a_{2} \\
a_{3}
\end{array}\right) ;\left(\begin{array}{ccc}
-\omega_{23}^{1} & \omega_{53}^{4} & \omega_{26}^{4} \\
\omega_{34}^{5} & -\omega_{31}^{2} & \omega_{61}^{5} \\
\omega_{42}^{6} & \omega_{15}^{6} & -\omega_{12}^{3}
\end{array}\right)=\left(\begin{array}{ccc}
b_{11} & b_{12} & b_{13} \\
b_{21} & b_{22} & b_{23} \\
b_{31} & b_{32} & b_{33}
\end{array}\right) .
$$

The Jacobi identities imply the twelve constraints

$$
\begin{aligned}
b_{i j} a_{j}+b_{j j} a_{i} & =0 \quad ; \quad i \neq j \\
b_{i k} b_{k j}+b_{k k} b_{i j} & =0 ; \quad i \neq j \neq k .
\end{aligned}
$$


Finally, the NS flux must satisfy the Bianchi identity $[12,6]$

$$
\omega \bar{H}_{3}=0
$$

with the contraction defined in (2.15). This constraint is satisfied automatically by the particular fluxes (2.10) and (2.16).

The superpotential induced by the fluxes can be obtained by performing the explicit Kaluza-Klein reduction $[4,6]$. The RR fluxes generate a superpotential only for the Kähler moduli, namely

$$
W_{K}=\int_{\mathrm{T}^{6}} e^{J_{c}} \wedge \bar{F}_{R R},
$$

where $\bar{F}_{R R}$ represents a formal sum of the even RR fluxes. NS and metric fluxes give a superpotential for the dilaton and complex structure moduli that can be cast as

$$
W_{Q}=\int_{\mathrm{T}^{6}} \Omega_{c} \wedge\left(\bar{H}_{3}+\omega J_{c}\right) .
$$

Recall that $\omega J_{c}$ is a 3 -form as defined in (2.15). Using previous results it is easy to compute $W_{Q}$. Combining with $W_{K}$ yields the full superpotential

$$
\begin{aligned}
W & =e_{0}+i h_{0} S+\sum_{i=1}^{3}\left[\left(i e_{i}-a_{i} S-b_{i i} U_{i}-\sum_{j \neq i} b_{i j} U_{j}\right) T_{i}-i h_{i} U_{i}\right] \\
& -q_{1} T_{2} T_{3}-q_{2} T_{1} T_{3}-q_{3} T_{1} T_{2}+i m T_{1} T_{2} T_{3} .
\end{aligned}
$$

This result was first presented in [6] and analyzed in detail in [8].

The fluxes also induce $\mathrm{RR}$ tadpoles. In this IIA orientifold there are $C_{7}$ tadpoles. In fact, the ten-dimensional action has a piece

$$
\int_{M_{4} \times \mathrm{T}^{6}}\left[C_{7} \wedge\left(m \bar{H}_{3}+\omega \bar{F}_{2}\right)\right]+\sum_{a} N_{a} \int_{M_{4} \times \Pi_{a}} C_{7} .
$$

The second term takes into account the coupling to O6-planes and stacks of D6-branes wrapping factorizable 3 -cycles

$$
\Pi_{a}=\left(n_{a}^{1}, m_{a}^{1}\right) \otimes\left(n_{a}^{2}, m_{a}^{2}\right) \otimes\left(n_{a}^{3}, m_{a}^{3}\right),
$$

and the corresponding orientifold images wrapping $\otimes_{i}\left(n_{a}^{i},-m_{a}^{i}\right)$. Here $n_{a}^{i}\left(m_{a}^{i}\right)$ are the wrapping numbers along the $x^{i}\left(y^{i}\right)$ torus directions. The O6-planes wrap $\otimes_{i}(1,0)$. From 
the component of $C_{7}$ along $x^{1}, x^{2}$ and $x^{3}$ we obtain

$$
\sum_{a} N_{a} n_{a}^{1} n_{a}^{2} n_{a}^{3}+\frac{1}{2}\left(m h_{0}+a_{1} q_{1}+a_{2} q_{2}+a_{3} q_{3}\right)=16 .
$$

From other components of $C_{7}$ there are further cancellation conditions

$$
\begin{aligned}
& \sum_{a} N_{a} n_{a}^{1} m_{a}^{2} m_{a}^{3}+\frac{1}{2}\left(m h_{1}-q_{1} b_{11}-q_{2} b_{21}-q_{3} b_{31}\right)=0 \\
& \sum_{a} N_{a} m_{a}^{1} n_{a}^{2} m_{a}^{3}+\frac{1}{2}\left(m h_{2}-q_{1} b_{12}-q_{2} b_{22}-q_{3} b_{32}\right)=0 \\
& \sum_{a} N_{a} m_{a}^{1} m_{a}^{2} n_{a}^{3}+\frac{1}{2}\left(m h_{3}-q_{1} b_{13}-q_{2} b_{23}-q_{3} b_{33}\right)=0 .
\end{aligned}
$$

In a $\mathbb{Z}_{2} \times \mathbb{Z}_{2}$ set-up there are other O6-planes that contribute -16 to the right hand side $[32,33]$.

\subsection{IIB orientifold with O3-planes}

We now discuss the IIB orientifold on $\mathrm{T}^{6} /\left[\Omega_{P}(-1)^{F_{L}} \sigma_{B}\right]$, with involution acting on the

Kähler form and the holomorphic 3-form as $\sigma_{B}(J)=J$ and $\sigma_{B}(\Omega)=-\Omega$. In terms of the coordinates this is $\sigma_{B}\left(x^{i}\right)=-x^{i}$ and $\sigma_{B}\left(y^{i}\right)=-y^{i}$. Thus, there are O3-planes that span space-time. Upon T-duality along $x^{1}, x^{2}$ and $x^{3}$, we recover the IIA orientifold with O6-branes of the previous section.

We have again seven diagonal closed moduli, the dilaton, three Kähler moduli and three complex structure moduli. We denote them as $S, T_{i}$ and $U_{i}$, even though they have different realizations in terms of the ten-dimensional degrees of freedom. In fact, the IIB and IIA moduli are related by T-duality as $T_{i} \leftrightarrow U_{i}$, whereas $S$ is invariant. The IIB complex structure fields are given directly by the toroidal complex structures, i.e. $U_{j}=\tau_{j}$. The complex dilaton is instead

$$
S=e^{-\phi}+i C_{0}
$$

where $C_{0}$ is the R-R 0 -form. The Kähler moduli can be extracted from the complexified 4-form

$$
\mathcal{J}_{c}=C_{4}+\frac{i}{2} e^{-\phi} J \wedge J=i \sum_{i=1}^{3} T_{i} \widetilde{\omega}_{i}
$$


where $C_{4}$ is the RR 4-form. The Kähler potential for the moduli has the same expression (2.9). The flux generated superpotential will be presented shortly.

The RR 3-form flux is odd under the orientifold involution. The most general flux can then be written as

$$
\overline{\mathcal{F}}_{3}=-m \alpha_{0}-e_{0} \beta_{0}+\sum_{i=1}^{3}\left(e_{i} \alpha_{i}-q_{i} \beta_{i}\right) .
$$

Observe that the flux coefficients are the same that appear in the RR IIA fluxes, c.f. (2.11). This is in agreement with T-duality. For the RR field strengths the Buscher rule states $[34,35]$

$$
F_{M N_{1} \cdots N_{p}} \stackrel{\mathrm{T}_{M}}{\longleftrightarrow} F_{N_{1} \cdots N_{p}}
$$

where $\mathrm{T}_{M}$ is T-duality in the $x^{M}$ direction. Then, performing T-dualities in $x^{1}, x^{2}, x^{3}$, in that order, on $\overline{\mathcal{F}}_{3}$ we obtain the IIA fluxes given in (2.11). Additional T-dualities in $x^{4}, x^{5}, x^{6}$, give the fluxes in the IIB orientifold on $\mathrm{T}^{6} / \Omega_{P}$ that has O9-planes. These results are summarized in table 1 .

\begin{tabular}{cccc} 
IIB/O3 & IIA/O6 & IIB/O9 & flux \\
\hline$\overline{\mathcal{F}}_{123}$ & $F_{0}$ & $-\boldsymbol{F}_{456}$ & $-m$ \\
$\overline{\mathcal{F}}_{423}$ & $F_{14}$ & $\boldsymbol{F}_{156}$ & $-q_{1}$ \\
$\overline{\mathcal{F}}_{153}$ & $F_{25}$ & $\boldsymbol{F}_{426}$ & $-q_{2}$ \\
$\overline{\mathcal{F}}_{126}$ & $F_{36}$ & $\boldsymbol{F}_{453}$ & $-q_{3}$ \\
$\overline{\mathcal{F}}_{156}$ & $F_{2536}$ & $-\boldsymbol{F}_{423}$ & $e_{1}$ \\
$\overline{\mathcal{F}}_{426}$ & $F_{1436}$ & $-\boldsymbol{F}_{153}$ & $e_{2}$ \\
$\overline{\mathcal{F}}_{453}$ & $F_{1425}$ & $-\boldsymbol{F}_{126}$ & $e_{3}$ \\
$\overline{\mathcal{F}}_{456}$ & $F_{142536}$ & $\boldsymbol{F}_{123}$ & $-e_{0}$
\end{tabular}

Table 1: RR IIB/O3 fluxes and their T-duals.

The NS 3-form flux is also odd under the orientifold involution. We thus have the general expansion

$$
\overline{\mathcal{H}}_{3}=h_{0} \beta_{0}-\sum_{i=1}^{3} a_{i} \alpha_{i}+\bar{h}_{0} \alpha_{0}-\sum_{i=1}^{3} \bar{a}_{i} \beta_{i} .
$$


For NS fluxes we can apply Buscher rules [34] to T-dualize when $\overline{\mathcal{H}}_{3}$ arises from a 2form independent of the dualized coordinates. In this case it is known that NS fluxes can transform into metric fluxes [36, 19]. As reviewed in [8], starting with $\overline{\mathcal{H}}_{3}=h_{0} \beta_{0}-\sum_{i} a_{i} \alpha_{i}$ and performing T-dualities in $x^{1}, x^{2}, x^{3}$, leads to the IIA fluxes

$$
\bar{H}_{3}=h_{0} \beta_{0} \quad ; \quad \omega_{56}^{1}=a_{1} \quad ; \quad \omega_{64}^{2}=a_{2} \quad ; \quad \omega_{45}^{3}=a_{3}
$$

If $\overline{\mathcal{H}}_{3} \sim \alpha_{0}$, the 2-form would depend on one the $x^{i}$. If $\overline{\mathcal{H}}_{3} \sim \beta_{i}$, one can still apply Buscher rules but they lead to more complicated geometries [19]. In [16] it was proposed that T-duality of the most general NS flux will lead to metric as well as to non-geometric fluxes. This will be the subject of next section.

In the IIB orientifold at hand there cannot be metric fluxes because the orientifold involution does not allow any even parameters $\omega_{M N}^{P}$.

The NS and RR 3-form fluxes induce the well-known superpotential [2]

$$
\mathcal{W}=\int_{\mathrm{T}^{6}}\left(\overline{\mathcal{F}}_{3}-i S \overline{\mathcal{H}}_{3}\right) \wedge \Omega .
$$

Substituting the fluxes we obtain

$$
\begin{aligned}
\mathcal{W} & =e_{0}+i \sum_{i=1}^{3} e_{i} U_{i}-q_{1} U_{2} U_{3}-q_{2} U_{1} U_{3}-q_{3} U_{1} U_{2}+i m U_{1} U_{2} U_{3} \\
& +S\left[i h_{0}-\sum_{i=1}^{3} a_{i} U_{i}+i \bar{a}_{1} U_{2} U_{3}+i \bar{a}_{2} U_{1} U_{3}+i \bar{a}_{3} U_{1} U_{2}-\bar{h}_{0} U_{1} U_{2} U_{3}\right]
\end{aligned}
$$

To go to type IIA we just exchange $U_{i} \leftrightarrow T_{i}$. Comparing with (2.21) clearly shows that the two superpotentials do not match. This hints at missing fluxes both in IIA and IIB. In the next section we will see that after including the non-geometric fluxes proposed in [16] the two superpotentials will be exact T-duals.

In this orientifold there is a $C_{4}$ tadpole induced by the fluxes. We know that it arises from the action term [37]

$$
\int_{M_{4} \times \mathrm{T}^{6}} C_{4} \wedge \overline{\mathcal{H}}_{3} \wedge \overline{\mathcal{F}}_{3}
$$

There are also contributions from O3-planes and a stack of $N_{\mathrm{D} 3}$ D3-branes. Substituting the fluxes and including the sources we obtain the tadpole cancellation condition

$$
N_{\mathrm{D} 3}+\frac{1}{2}\left[m h_{0}-e_{0} \bar{h}_{0}+\sum_{i}\left(q_{i} a_{i}+e_{i} \bar{a}_{i}\right)\right]=16 .
$$


Upon T-duality this agrees with the $C_{7}$ tadpole (2.24). We also expect $C_{8}$ tadpoles to match (2.25) but they cannot be induced by RR and NS fluxes alone, clearly some terms are missing. This is another indication that non-geometric fluxes are required by T-duality.

\section{T-duality and non-geometric fluxes}

There are two types of non-geometric fluxes introduced in [16], the tensors $Q_{P}^{M N}$ and $R^{M N P}$ that are completely antisymmetric in the upper indices. The $Q$ 's are odd under the orientifold involution, while the $R$ 's are even. Recall that $\bar{H}_{M N P}$ is odd and $\omega_{N P}^{M}$ is even. Now, the crucial property is that all these fluxes are related by T-duality according to the chain

$$
-\bar{H}_{M N P} \stackrel{\mathrm{T}_{M}}{\longleftrightarrow} \omega_{N P}^{M} \stackrel{\mathrm{T}_{N}}{\longleftrightarrow} Q_{P}^{M N} \stackrel{\mathrm{T}_{P}}{\longleftrightarrow}-R^{M N P}
$$

We have introduced some extra signs in order to agree with the conventions used in [8]. For example, we have seen that $\bar{H}_{156}=-a_{1}$ transforms under $\mathrm{T}_{1}$-duality into $\omega_{56}^{1}=a_{1}$.

In IIB with O3-planes there are neither metric fluxes nor non-geometric fluxes of type $R$ because there are no such tensors even under the orientifold involution. There are only odd non-geometric fluxes, denoted $\mathcal{Q}_{P}^{M N}$, that comprise twenty-four free parameters taking each index in a different sub-torus. There are also odd NS fluxes $\overline{\mathcal{H}}$.

In IIA with O6-planes there are non-geometric fluxes $Q$ and $R$, as well as NS $\bar{H}$ and metric fluxes $\omega$. It is also interesting to consider IIB with O9-planes in which the orientifold involution is the identity. In this case there can only be even fluxes denoted $\boldsymbol{\omega}$ and $\boldsymbol{R}$.

Using the rule (3.1) and starting with the NS and metric fluxes in the IIA side we can perform a chain of $\mathrm{T}_{1}, \mathrm{~T}_{2}$ and $\mathrm{T}_{3}$ dualities, i.e. mirror symmetry, to obtain the corresponding fluxes in IIB. For example, under mirror symmetry, $\bar{H}_{i j c} \rightarrow-\mathcal{Q}_{c}^{i j}, \omega_{j k}^{i} \rightarrow$ $\mathcal{Q}_{i}^{j k}$, and so on. The results are summarized in table 2. Notice that the indices are ordered cyclically according to the sub-torus to which they belong. The IIB/O9 fluxes are obtained from IIB/O3 by performing six T-dualities, $\mathrm{T}_{1}, \cdots, \mathrm{T}_{6}$, or obviously from IIA/O6 by applying only $\mathrm{T}_{4}, \mathrm{~T}_{5}, \mathrm{~T}_{6}$. 


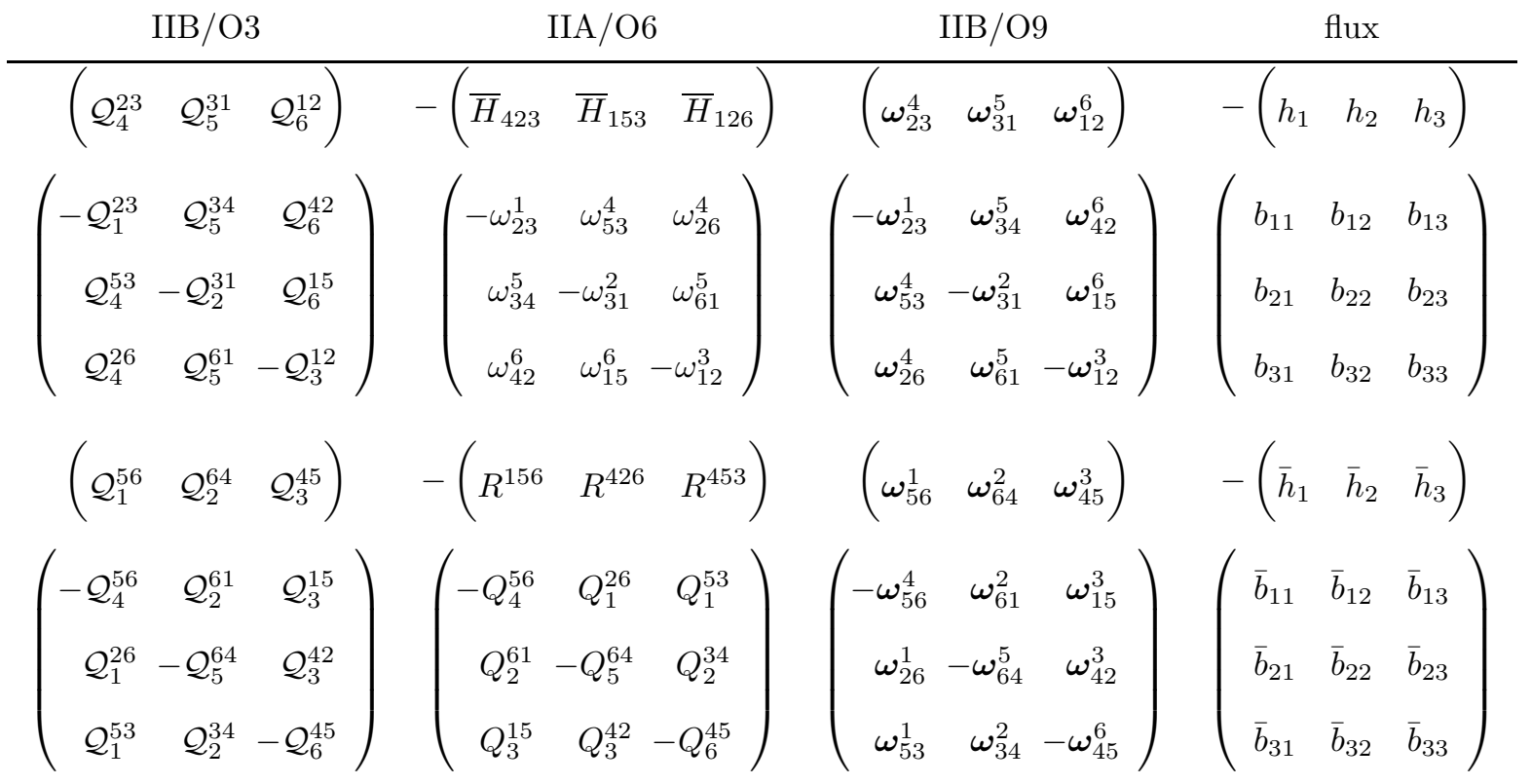

Table 2: Non-geometric IIB/O3 fluxes and their T-duals.

So far we have accounted for twelve non-geometric IIB/O3 fluxes, those related to the IIA/O6 backgrounds $h_{i}$ (NS) and $b_{i j}$ (metric). The IIB/O3 orientifold projection still allows another twelve components for $\mathcal{Q}$, denoted $\bar{h}_{i}$ and $\bar{b}_{i j}$ as shown in table 2. Applying T-dualities we then obtain the corresponding fluxes in IIA/O6 and IIB/O9. These results are also displayed in table 2 .

Finally, applying T-duality to the IIB/O3 NS fluxes reveals some non-geometric fluxes in IIA/O6 and IIB/O9. For example, acting with the chain of $\mathrm{T}_{1}, \mathrm{~T}_{2}$ and $\mathrm{T}_{3}$ dualities gives $\overline{\mathcal{H}}_{123} \rightarrow R^{123}, \overline{\mathcal{H}}_{423} \rightarrow-Q_{4}^{23}$, etc.. We have already seen that the $\overline{\mathcal{H}}_{i b c}$ are T-dual to IIA metric fluxes. These results are collected in table 3. We now have a complete explicit dictionary to translate from one orientifold to another.

The next task is to determine the superpotential and tadpoles induced by the nongeometric fluxes. In sections 3.1 and 3.2 we consider type IIB with O3-planes and type IIA with O6-planes to some extent. We will give explicit expressions for the superpotentials as integrals involving the flux tensors and the complexified forms that encode the moduli. Tadpoles of RR $C_{p}$ forms are written in terms of the flux combinations that couple to them. The IIB orientifold with O9-planes will be briefly surveyed. The NS, metric and non-geometric fluxes are expected to satisfy Bianchi identities that generalize (2.14) and 


\begin{tabular}{cccc} 
IIB/O3 & IIA/O6 & IIB/O9 & flux \\
\hline$\overline{\mathcal{H}}_{123}$ & $R^{123}$ & $\boldsymbol{R}^{123}$ & $\bar{h}_{0}$ \\
$\overline{\mathcal{H}}_{423}$ & $-Q_{4}^{23}$ & $\boldsymbol{R}^{423}$ & $-\bar{a}_{1}$ \\
$\overline{\mathcal{H}}_{153}$ & $-Q_{5}^{31}$ & $\boldsymbol{R}^{153}$ & $-\bar{a}_{2}$ \\
$\overline{\mathcal{H}}_{126}$ & $-Q_{6}^{12}$ & $\boldsymbol{R}^{126}$ & $-\bar{a}_{3}$ \\
$\overline{\mathcal{H}}_{156}$ & $-\omega_{56}^{1}$ & $\boldsymbol{R}^{156}$ & $-a_{1}$ \\
$\overline{\mathcal{H}}_{426}$ & $-\omega_{64}^{2}$ & $\boldsymbol{R}^{426}$ & $-a_{2}$ \\
$\overline{\mathcal{H}}_{453}$ & $-\omega_{45}^{3}$ & $\boldsymbol{R}^{453}$ & $-a_{3}$ \\
$\overline{\mathcal{H}}_{456}$ & $\bar{H}_{456}$ & $\boldsymbol{R}^{456}$ & $h_{0}$
\end{tabular}

Table 3: NS IIB/O3 fluxes and their T-duals.

(2.18). This type of constraints will be derived in section 3.4.

With the T-dual superpotential available, the next step is to analyze the moduli potential. In section 3.5 we will discuss some classes of vacua and compare with previous results.

\subsection{T-dual superpotential and tadpoles in IIB with O3-planes}

We want to determine the superpotential and tadpoles induced by the $\mathcal{Q}$ fluxes. An useful result is that we can contract a $p$-form $\mathcal{X}$ with $\mathcal{Q}$ to obtain a $(p-1)$-form $\mathcal{Q} \mathcal{X}$ with components

$$
(\mathcal{Q X})_{L M_{1} \cdots M_{p-2}}=\frac{1}{2} \mathcal{Q}_{[L}^{A B} \mathcal{X}_{\left.M_{1} \cdots M_{p-2}\right] A B}
$$

This is analogous to the contraction with $\omega$ defined in (2.15).

Observing the IIA result (2.21) it is clear that the $\mathcal{Q}$ fluxes must induce new terms linear in the $T_{i}$ and up to cubic order in the $U_{i}$. Such terms can be generated by adding to $\mathcal{W}$ a piece $\int \mathcal{Q}_{\mathcal{J}} \wedge \Omega$, where $\mathcal{J}_{c}$ is the 4 -form that encodes the Kähler moduli, c.f. (2.27), and $\mathcal{Q J}_{c}$ is a 3 -form according to (3.2). The complete IIB superpotential is then

$$
\mathcal{W}=\int_{\mathrm{T}^{6}}\left(\overline{\mathcal{F}}_{3}-i S \overline{\mathcal{H}}_{3}+\mathcal{Q} \mathcal{J}_{c}\right) \wedge \Omega
$$


Substituting the fluxes yields

$$
\begin{aligned}
\mathcal{W} & =e_{0}+i \sum_{i=1}^{3} e_{i} U_{i}-q_{1} U_{2} U_{3}-q_{2} U_{1} U_{3}-q_{3} U_{1} U_{2}+i m U_{1} U_{2} U_{3} \\
& +S\left[i h_{0}-\sum_{i=1}^{3} a_{i} U_{i}+i \bar{a}_{1} U_{2} U_{3}+i \bar{a}_{2} U_{1} U_{3}+i \bar{a}_{3} U_{1} U_{2}-\bar{h}_{0} U_{1} U_{2} U_{3}\right] \\
& +\sum_{i=1}^{3} T_{i}\left[-i h_{i}-\sum_{j=1}^{3} U_{j} b_{j i}+i U_{2} U_{3} \bar{b}_{1 i}+i U_{1} U_{3} \bar{b}_{2 i}+i U_{1} U_{2} \bar{b}_{3 i}+U_{1} U_{2} U_{3} \bar{h}_{i}\right] .
\end{aligned}
$$

The $\mathcal{Q}$-induced terms are in the last row.

The general superpotential agrees with the proposal of [16] if we assume a symmetry under exchange of the three sub-tori. This amounts to setting $T_{i}=T$ and $U_{i}=U$ together with the choice of fluxes

$$
\begin{aligned}
& e_{i}=e \quad ; \quad q_{i}=q \quad ; \quad a_{i}=a \quad ; \quad \bar{a}_{i}=\bar{a} \quad ; \quad h_{i}=h \quad ; \quad \bar{h}_{i}=\bar{h}_{i} ; \\
& b_{i j}=b(i \neq j) \quad ; \quad b_{i i}=\beta \quad ; \quad \bar{b}_{i j}=\bar{b}(i \neq j) \quad ; \quad \bar{b}_{i i}=\bar{\beta} .
\end{aligned}
$$

We have also taken $b_{i j}=b_{j i}$ and $\bar{b}_{i j}=\bar{b}_{j i}$. The superpotential then reduces to

$$
\begin{aligned}
\mathcal{W} & =e_{0}+3 i e U-3 q U^{2}+i m U^{3} \\
& +S\left[i h_{0}-3 a U+3 i \bar{a} U^{2}-\bar{h}_{0} U^{3}\right] \\
& +3 T\left[-i h-(2 b+\beta) U+i(2 \bar{b}+\bar{\beta}) U^{2}+\bar{h} U^{3}\right] .
\end{aligned}
$$

The fluxes that enter in the superpotential must satisfy some tadpole cancellation conditions and Bianchi constraints that will be examined in the next sections.

In this orientifold there is a $C_{4}$ tadpole already discussed in section 2.3. We also expect $C_{8}$ tadpoles that can receive contributions from D7-branes and O7-planes. The flux piece must be a 2 -form suitable to wedge with $C_{8}$. A natural candidate is $\mathcal{Q} \overline{\mathcal{F}}_{3}$, where the 2 -form is computed according to (3.2). The proposal for the $C_{8}$ tadpole is just

$$
-\int_{M_{4} \times \mathrm{T}^{6}} C_{8} \wedge \mathcal{Q} \overline{\mathcal{F}}_{3}
$$

The minus sign in front is needed to match the known IIA results when only NS and metric fluxes are present. There are three different tadpoles according to the components 
of $C_{8}$ that can couple to $\mathrm{D} 7_{i}$-branes. As usual, a $\mathrm{D} 7_{i}$-brane is transverse to $\mathrm{T}_{i}^{2}$ while wrapping $\mathrm{T}_{j}^{2}$ and $\mathrm{T}_{k}^{2}, i \neq j \neq k$. For example, the flux contribution to the $\mathrm{D}_{1}$ tadpole comes from

$$
\left(\mathcal{Q} \overline{\mathcal{F}}_{3}\right)_{14}=-m h_{1}+e_{0} \bar{h}_{1}+\sum_{i}\left(q_{i} b_{i 1}+e_{i} \bar{b}_{i 1}\right) \text {. }
$$

Taking into account a number $N_{\mathrm{D} 7_{i}}$ of $\mathrm{D} 7_{i}$-branes, and the flux tadpoles arising from (3.7), gives the cancellation conditions

$$
-N_{\mathrm{D} 7_{i}}+\frac{1}{2}\left[m h_{i}-e_{0} \bar{h}_{i}-\sum_{j}\left(q_{j} b_{j i}+e_{j} \bar{b}_{j i}\right)\right]=0 .
$$

We have not included O7-planes, absent in a setup without $\mathbb{Z}_{2} \times \mathbb{Z}_{2}$ orbifolding. A new interesting feature is the dependence of the tadpoles on all RR fluxes.

\subsection{T-dual superpotential and tadpoles in IIA with O6-planes}

In this case there are non-geometric $Q$ and $R$ fluxes. As in (3.2), we can contract $Q$ with a $p$-form $\mathcal{X}$ to obtain a $(p-1)$-form $Q \mathcal{X}$. Analogously, contracting with $R$ we obtain a $(p-3)$-form with components

$$
(R \mathcal{X})_{M_{1} \cdots M_{p-3}}=\frac{1}{6} R^{A B C} \mathcal{X}_{\left[M_{1} \cdots M_{p-3}\right] A B C}
$$

For example, the 3 -form $R \bar{F}_{6}$ contributes to $C_{7}$ tadpoles.

The $Q$ and $R$ fluxes are expected to induce superpotential terms quadratic and cubic in the IIA Kähler moduli. There are appropriate 2 and 3 -forms that encode the required combination of the $T_{i}$, namely

$$
\begin{aligned}
& J_{c}^{(2)} \equiv \frac{1}{2} J_{c} \wedge J_{c}=-T_{2} T_{3} \widetilde{\omega}_{1}-T_{1} T_{3} \widetilde{\omega}_{2}-T_{1} T_{2} \widetilde{\omega}_{3} \\
& J_{c}^{(3)} \equiv \frac{1}{6} J_{c} \wedge J_{c} \wedge J_{c}=-i T_{1} T_{2} T_{3} \alpha_{0} \wedge \beta_{0} .
\end{aligned}
$$

Then, the IIA superpotential T-dual to (3.3) can be written as

$$
W=\int_{\mathrm{T}^{6}}\left[e^{J_{c}} \wedge \bar{F}_{R R}+\Omega_{c} \wedge\left(\bar{H}_{3}+\omega J_{c}+Q J_{c}^{(2)}+R J_{c}^{(3)}\right)\right] .
$$

Substituting the fluxes precisely reproduces (3.4) upon exchanging $T_{i} \leftrightarrow U_{i}$. 
The idea behind the general formula for $W$ is to wedge $\Omega_{c}$ with all available 3 -forms. An analogous reasoning suggests that the $C_{7}$ tadpoles due to all fluxes follow from

$$
\int_{M_{4} \times \mathrm{T}^{6}} C_{7} \wedge\left(-\bar{H}_{3} \bar{F}_{0}+\omega \bar{F}_{2}-Q \bar{F}_{4}+R \bar{F}_{6}\right) .
$$

The signs have been chosen to match results in type IIB. Including tadpoles due to O6planes and stacks of intersecting D6-branes leads to the general cancellation conditions

$$
\begin{aligned}
\sum_{a} N_{a} n_{a}^{1} n_{a}^{2} n_{a}^{3}+\frac{1}{2}\left[m h_{0}-e_{0} \bar{h}_{0}+\sum_{i}\left(q_{i} a_{i}+e_{i} \bar{a}_{i}\right)\right] & =16, \\
\sum_{a} N_{a} n_{a}^{1} m_{a}^{2} m_{a}^{3}+\frac{1}{2}\left[m h_{1}-e_{0} \bar{h}_{1}-\sum_{i}\left(q_{i} b_{i 1}+e_{i} \bar{b}_{i 1}\right)\right] & =0 \\
\sum_{a} N_{a} m_{a}^{1} n_{a}^{2} m_{a}^{3}+\frac{1}{2}\left[m h_{2}-e_{0} \bar{h}_{2}-\sum_{i}\left(q_{i} b_{i 2}+e_{i} \bar{b}_{i 2}\right)\right] & =0 \\
\sum_{a} N_{a} m_{a}^{1} m_{a}^{2} n_{a}^{3}+\frac{1}{2}\left[m h_{3}-e_{0} \bar{h}_{3}-\sum_{i}\left(q_{i} b_{i 3}+e_{i} \bar{b}_{i 3}\right)\right] & =0 .
\end{aligned}
$$

These agree with (2.35) and (3.9).

\subsection{T-dual superpotential and tadpoles in IIB with O9-planes}

In this case the orientifold action is only $\Omega_{P}$. Since the orientifold involution is the identity only even fluxes are allowed. There are eight $\operatorname{RR} \boldsymbol{F}_{L M N}$, twenty-four metric $\boldsymbol{\omega}_{M N}^{L}$, and eight non-geometric $\boldsymbol{R}^{L M N}$. The components are displayed in tables 1, 2 and 3 .

The superpotential can be derived from IIB/O3 results by implementing T-dualities in each of the six internal coordinates. The moduli then transform as $S \leftrightarrow S, T_{i} \leftrightarrow T_{i}$, but $U_{i} \leftrightarrow 1 / U_{i}$. The Kähler potential transforms as

$$
K \rightarrow K+\log \left|U_{1} U_{2} U_{3}\right|^{2}
$$

Invariance of the Kähler function, $\mathcal{G}=K+\log |\mathcal{W}|^{2}$, then requires

$$
W_{\mathrm{O} 9}=\frac{-i \mathcal{W}}{U_{1} U_{2} U_{3}}
$$

where we have chosen a convenient phase. Therefore, in terms of IIB/O9 moduli,

$$
W_{\mathrm{O} 9}=m+i \sum_{i=1}^{3} q_{i} U_{i}+e_{1} U_{2} U_{3}+e_{2} U_{1} U_{3}+e_{3} U_{1} U_{2}-i e_{0} U_{1} U_{2} U_{3}
$$




$$
\begin{aligned}
& +S\left[i \bar{h}_{0}+\sum_{i=1}^{3} \bar{a}_{i} U_{i}+i a_{1} U_{2} U_{3}+i a_{2} U_{1} U_{3}+i a_{3} U_{1} U_{2}+h_{0} U_{1} U_{2} U_{3}\right] \\
& +\sum_{i=1}^{3} T_{i}\left[-i \bar{h}_{i}+\sum_{j=1}^{3} \bar{b}_{j i} U_{j}+i b_{1 i} U_{2} U_{3}+i b_{2 i} U_{1} U_{3}+i b_{3 i} U_{1} U_{2}-h_{i} U_{1} U_{2} U_{3}\right]
\end{aligned}
$$

In absence of metric $\boldsymbol{\omega}$ and non-geometric $\boldsymbol{R}$ fluxes $W_{\text {O9 }}$ depends only on the complex structure moduli. Linear terms in $T_{i}$ and $S$ are induced by $\boldsymbol{\omega}$ and $\boldsymbol{R}$ respectively.

Now there are O9-planes and we can add D9-branes. We then anticipate that fluxes contribute to a $C_{10}$ tadpole. Indeed, there is a candidate tadpole term

$$
\int_{M_{4} \times \mathrm{T}^{6}} C_{10} \wedge \boldsymbol{R F _ { 3 }}
$$

where $\boldsymbol{R F}_{3}$ is a 0 -form according to (3.10). Substituting the fluxes and including sources gives

$$
N_{\mathrm{D} 9}+\frac{1}{2}\left[m h_{0}-e_{0} \bar{h}_{0}+\sum_{i}\left(q_{i} a_{i}+e_{i} \bar{a}_{i}\right)\right]=16 .
$$

To match the IIB/O3 results there must also be $C_{6}$ tadpoles. With the available fluxes we can indeed have a term

$$
\int_{M_{4} \times \mathrm{T}^{6}} C_{6} \wedge \boldsymbol{\omega} \boldsymbol{F}_{3}
$$

where $\boldsymbol{\omega} \boldsymbol{F}_{3}$ is a 4 -form according to $(2.15)$. We can also add D $5_{i}$-branes that wrap $\mathrm{T}_{i}^{2}$. We then find cancellation conditions

$$
N_{\mathrm{D} 5_{i}}+\frac{1}{2}\left[m h_{i}-e_{0} \bar{h}_{i}-\sum_{j}\left(q_{j} b_{j i}+e_{j} \bar{b}_{j i}\right)\right]=0 .
$$

We have not included O5-planes.

\subsection{Constraints on NS, metric and non-geometric fluxes}

We saw in chapter 2 that the geometric fluxes $\omega_{N P}^{M}$ are subject to the Bianchi identities in eq.(2.14). To find the analogous constraints for non-geometric fluxes we will follow the approach of [16], see also [25]. The strategy is to extend the algebra of isometry

generators $Z_{M}$ to include generators $X^{M}, M=1, \cdots, 6$. The $X^{M}$ are associated to gauge symmetries arising from reduction of the $B$-field on $\mathrm{T}^{6}$ with fluxes [12]. The extended 
algebra has the NS, metric and non-geometric fluxes as structure constants. The most general algebra is then

$$
\begin{aligned}
{\left[Z_{M}, Z_{N}\right] } & =-\bar{H}_{M N P} X^{P}+\omega_{M N}^{P} Z_{P} \\
{\left[Z_{M}, X^{P}\right] } & =-\omega_{M N}^{P} X^{N}+Q_{M}^{P R} Z_{R} \\
{\left[X^{M}, X^{N}\right] } & =Q_{P}^{M N} X^{P}-R^{M N P} Z_{P} .
\end{aligned}
$$

The Jacobi identities of the algebra give constraints on the fluxes ${ }^{1}$.

The proposed algebra actually applies to any of the IIA or IIB orientifolds, provided that all fluxes allowed by the orientifold action are kept in each case. In fact, using (3.1) and

$$
Z_{M} \stackrel{\mathrm{T}_{M}}{\longleftrightarrow} X^{M}
$$

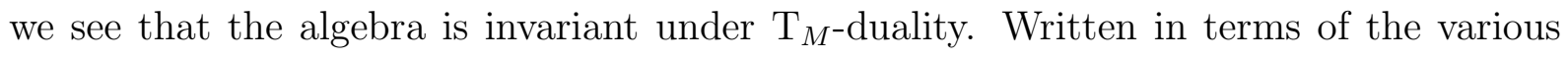
tensors, the Jacobi identities take a different form in each case. However, in terms of the individual flux parameters that appear in the T-dual superpotential there is just one set of constraints valid on all orientifolds.

It is convenient to work with the IIB with O3-planes in which only NS $\overline{\mathcal{H}}$ and nongeometric $\mathcal{Q}$ fluxes appear. The $Z Z Z$ Jacobi identity leads to

$$
\mathcal{Q}_{[L}^{R P} \overline{\mathcal{H}}_{M N] P}=0
$$

Substituting the fluxes in tables 2 and 3 then yields

$$
\begin{aligned}
\bar{h}_{0} h_{j}+\bar{a}_{i} b_{i j}+\bar{a}_{j} b_{j j}-a_{k} \bar{b}_{k j} & =0, \\
h_{0} \bar{h}_{j}+a_{i} \bar{b}_{i j}+a_{j} \bar{b}_{j j}-\bar{a}_{k} b_{k j} & =0, \\
\bar{h}_{0} b_{k j}+\bar{a}_{i} \bar{b}_{j j}+\bar{a}_{j} \bar{b}_{i j}-a_{k} \bar{h}_{j} & =0, \\
h_{0} \bar{b}_{k j}+a_{i} b_{j j}+a_{j} b_{i j}-\bar{a}_{k} h_{j} & =0 .
\end{aligned}
$$

In all cases $i \neq j \neq k$. The $X X X$ Jacobi identity simply gives

$$
\mathcal{Q}_{P}^{[M N} \mathcal{Q}_{R}^{L] P}=0
$$

\footnotetext{
${ }^{1}$ In contrast to the algebras considered in [13], here there are always six $Z_{M}$ and six $X^{M}$ generators, as required to account for all fluxes.
} 
In terms of the explicit fluxes

$$
\begin{aligned}
-b_{i i} b_{j k}+\bar{b}_{k i} h_{k}+h_{i} \bar{b}_{k k}-b_{j i} b_{i k} & =0, \\
-\bar{b}_{i i} \bar{b}_{j k}+b_{k i} \bar{h}_{k}+\bar{h}_{i} b_{k k}-\bar{b}_{j i} \bar{b}_{i k} & =0, \\
-b_{i i} \bar{b}_{i j}+\bar{b}_{j i} b_{j j}+h_{i} \bar{h}_{j}-b_{k i} \bar{b}_{k j} & =0, \\
\bar{b}_{i i} b_{i j}-b_{j i} \bar{b}_{j j}+h_{i} \bar{h}_{j}-b_{k i} \bar{b}_{k j} & =0 .
\end{aligned}
$$

In all cases $i \neq j \neq k$. There are no further constraints from other Jacobi identities.

With the isotropic fluxes given in (3.5) the constraints read

$$
\begin{aligned}
\bar{h}_{0} h+\bar{a}(b+\beta)-a \bar{b} & =0, \\
h_{0} \bar{h}+a(\bar{b}+\bar{\beta})-\bar{a} b & =0, \\
\bar{h}_{0} b+\bar{a}(\bar{b}+\bar{\beta})-a \bar{h} & =0, \\
h_{0} \bar{b}+a(b+\beta)-\bar{a} h & =0, \\
h(\bar{b}+\bar{\beta})-b(b+\beta) & =0, \\
\bar{h}(b+\beta)-\bar{b}(\bar{b}+\bar{\beta}) & =0, \\
h \bar{h}-b \bar{b} & =0 .
\end{aligned}
$$

Some classes of solutions are:

$$
\begin{aligned}
& \text { 1. } h=\bar{h}=b=\beta=\bar{b}=\bar{\beta}=0(\mathcal{Q}=0) ; a, \bar{a}, h_{0}, \bar{h}_{0} \neq 0(\overline{\mathcal{H}} \neq 0) \\
& \text { 2. } a=\bar{a}=h_{0}=\bar{h}_{0}=0(\overline{\mathcal{H}}=0) ; h \bar{h}=b \bar{b} ; h(\bar{b}+\bar{\beta})=b(b+\beta) . \\
& \text { 3. } a=\bar{a}=h=\bar{h}=b=\bar{b}=0 ; h_{0}, \bar{h}_{0}, \beta, \bar{\beta} \neq 0 . \\
& \text { 4. } \beta=-b \quad ; \quad \bar{\beta}=-\bar{b} ; h \bar{h}=b \bar{b} ; \bar{h}_{0} h=a \bar{b} \quad ; \quad h_{0} \bar{h}=\bar{a} b
\end{aligned}
$$

\subsection{Some vacua with T-dual fluxes}

In this section we work in the IIA/O6 setup for ease of comparison with results of [8]. Our purpose is to see the effect of the new non-geometric fluxes in some simple examples. 
We focus on no-scale type of superpotentials depending only on four moduli. In this case the scalar potential is positive definite and is minimized with respect to all fields when the four covariant derivatives of $W$ vanish.

To be concrete we consider the moduli $S, U_{1}, T_{2}$ and $T_{3}$. Turning on all fluxes visible to these fields gives the generic superpotential

$$
\begin{aligned}
W & =e_{0}+i e_{2} T_{2}+i e_{3} T_{3}-q_{1} T_{2} T_{3}+S\left[i h_{0}-a_{2} T_{2}-a_{3} T_{3}+i \bar{a}_{1} T_{2} T_{3}\right] \\
& -U_{1}\left[i h_{1}+b_{21} T_{2}+b_{31} T_{3}-i \bar{b}_{11} T_{2} T_{3}\right]
\end{aligned}
$$

It is easily proven that the set of fluxes in $W$ satisfies the Bianchi identities of the previous section. Moreover, these fluxes do not contribute to tadpoles.

When one of the non-geometric flux parameters is zero we can map $W$ to one of the cases studied in [8]. For instance, when $\bar{a}_{1}=0$, redefining $U_{1} \rightarrow T_{1}$, and relabelling fluxes appropriately, brings us to the NS-3 example of [8]. If $a_{2}=a_{3}=0$, we can compare with the simpler NS-1 model. In this case we conclude that there are minima only if $h_{0} \neq 0$, $\bar{b}_{11} \neq 0$ and furthermore

$$
h_{1} \bar{b}_{11}=b_{31} b_{21} \quad ; \quad e_{2} \bar{b}_{11}=-q_{1} b_{21} \quad ; \quad e_{3} \bar{b}_{11}=-q_{1} b_{31}
$$

We also find that axions are fixed but the real parts of moduli remain undetermined except for a relation $h_{0} \operatorname{Re} S=\bar{b}_{11} \operatorname{Re} U_{1} \operatorname{Re} T_{2} \operatorname{Re} T_{3}$. The situation with $\bar{b}_{11}=0$ also corresponds to the NS-3 example [8]. It is only when $\bar{a}_{1} \neq 0$ and $\bar{b}_{11} \neq 0$, so that both cubic terms are present in $W$, that we can have a different kind of no-scale model.

We have analyzed the case with both non-geometric fluxes turned on to some extent. To simplify we choose $a_{i}=a, b_{i 1}=b$ and $e_{i}=e$, which allows vacua with $T_{2}=T_{3}=T$. Some generic results can be extracted. For instance,

$$
\begin{aligned}
\bar{a} \operatorname{Im} S+\bar{b} \operatorname{Im} U & =-q \\
(a+\bar{a} \operatorname{Im} T) \operatorname{Re} S & =(b+\bar{b} \operatorname{Im} T) \operatorname{Re} U
\end{aligned}
$$

where we have dropped subindices. There are more equations to be solved. In general only the ratio of $\operatorname{Re} S$ and $\operatorname{Re} U$ is fixed. When $a \bar{b} \neq b \bar{a}$, the fluxes generically determine all axions as well as $\operatorname{Re} T$. When $a \bar{b}=b \bar{a}$, and $e \bar{a}=-q a$ by consistency, there are 
two types of solutions. In one type, with $h q \neq-e b$, all axions are determined, in fact $\operatorname{Im} T=-a / \bar{a}$, and $(\operatorname{Re} T)^{4}$ is completely fixed in terms of fluxes alone. In the other type, with fluxes further satisfying $h e=-e_{0} b$ and $h_{0} b=-h a$, all axions are undetermined and $(\operatorname{Re} T)^{2}$ can only be given in terms of $\operatorname{Im} T$. For instance by choosing the fluxes $a=\bar{a}=b=\bar{b}=h_{0}=e_{0}=e=-q=2$ and $h=0$ we find the solution

$$
\operatorname{Im} S=-\operatorname{Im} T=1 \quad ; \quad \operatorname{Im} U=0 \quad ; \quad \operatorname{Re} T=2^{\frac{1}{4}} \quad ; \quad \operatorname{Re} U=\sqrt{2} \operatorname{Re} S .
$$

Taking larger fluxes it should be possible to obtain larger $\operatorname{Re} T$.

To summarize, adding non-geometric backgrounds leads to new no-scale vacua, inequivalent to those with only RR, NS and metric fluxes. However, the examples that we have examined have qualitative properties analogous to the vacua analyzed in [8]. In section 5 we will briefly consider Minkowski vacua in presence of non-geometric fluxes.

\section{IIB S-duality and fluxes}

We know that type IIB string theory is S-duality invariant. Upon the orientifold compactification here considered we still expect the theory to reflect this underlying invariance. In fact, in the absence of non-geometric fluxes, when only $\overline{\mathcal{H}}_{3}$ and $\overline{\mathcal{F}}_{3}$ are present, the theory is explicitly S-duality invariant because these fluxes transform appropriately. On

the other hand, once we have added non-geometric fluxes $\mathcal{Q}$ the theory does not respect S-duality. We will see in this chapter that S-duality invariance requires the presence of extra flux degrees of freedom $\mathcal{P}$.

We want to implement invariance under the $S L(2, \mathbb{Z})$ S-duality transformations

$$
S \rightarrow \frac{k S-i \ell}{i m S+n} \quad ; \quad k n-\ell m=1 \quad ; \quad k, \ell, m, n \in \mathbb{Z} .
$$

The factors of $i$ are needed since in our conventions $\operatorname{Re} S=1 / g_{s}$. The Kähler potential, $K=-\log \left(S+S^{*}\right)+\cdots$, transforms as

$$
K \rightarrow K+\log |i m S+n|^{2} .
$$

Thus, the Kähler function, $\mathcal{G}=K+\log |\mathcal{W}|^{2}$, is invariant provided the superpotential verifies

$$
\mathcal{W} \rightarrow \frac{\mathcal{W}}{i m S+n}
$$


With only NS and RR fluxes turned on this follows simply because under S-duality the NS and RR 3-forms transform as

$$
\left(\begin{array}{l}
\overline{\mathcal{F}}_{3} \\
\overline{\mathcal{H}}_{3}
\end{array}\right) \rightarrow\left(\begin{array}{ll}
k & \ell \\
m & n
\end{array}\right)\left(\begin{array}{l}
\overline{\mathcal{F}}_{3} \\
\overline{\mathcal{H}}_{3}
\end{array}\right)
$$

In particular, when $S \rightarrow 1 / S, \overline{\mathcal{F}}_{3} \rightarrow-\overline{\mathcal{H}}_{3}$ and $\overline{\mathcal{H}}_{3} \rightarrow \overline{\mathcal{F}}_{3}$.

The question is now how to maintain S-duality after including the non-geometric fluxes $\mathcal{Q}$. To obtain a full S-dual superpotential we simply propose to add a new set of fluxes, denoted $\mathcal{P}$, with the same tensor structure and number of components as $\mathcal{Q}$. Concretely, we conjecture that the superpotential is given by

$$
\mathcal{W}=\int_{\mathrm{T}^{6}}\left[\left(\overline{\mathcal{F}}_{3}-i S \overline{\mathcal{H}}_{3}\right)+(\mathcal{Q}-i S \mathcal{P}) \mathcal{J}_{c}\right] \wedge \Omega
$$

The action will be invariant as long as $\mathcal{Q}$ and $\mathcal{P}$ fluxes transform as

$$
\left(\begin{array}{l}
\mathcal{Q} \\
\mathcal{P}
\end{array}\right) \rightarrow\left(\begin{array}{ll}
k & \ell \\
m & n
\end{array}\right)\left(\begin{array}{l}
\mathcal{Q} \\
\mathcal{P}
\end{array}\right)
$$

In particular, when $S \rightarrow 1 / S$, one has $\mathcal{Q} \rightarrow-\mathcal{P}$ and $\mathcal{P} \rightarrow \mathcal{Q}$.

The new objects $\mathcal{P}_{P}^{M N}$ are some sort of RR non-geometric fluxes. For the components we use the notation

$$
\left(\begin{array}{l}
\mathcal{P}_{4}^{23} \\
\mathcal{P}_{5}^{31} \\
\mathcal{P}_{6}^{12}
\end{array}\right)=\left(\begin{array}{c}
-f_{1} \\
-f_{2} \\
-f_{3}
\end{array}\right) ; \quad\left(\begin{array}{ccc}
-\mathcal{P}_{1}^{23} & \mathcal{P}_{5}^{34} & \mathcal{P}_{6}^{42} \\
\mathcal{P}_{4}^{53} & -\mathcal{P}_{2}^{31} & \mathcal{P}_{6}^{15} \\
\mathcal{P}_{4}^{26} & \mathcal{P}_{5}^{61} & -\mathcal{P}_{3}^{12}
\end{array}\right)=\left(\begin{array}{ccc}
g_{11} & g_{12} & g_{13} \\
g_{21} & g_{22} & g_{23} \\
g_{31} & g_{32} & g_{33}
\end{array}\right)
$$

together with

$$
\left(\begin{array}{l}
\mathcal{P}_{1}^{56} \\
\mathcal{P}_{2}^{64} \\
\mathcal{P}_{3}^{45}
\end{array}\right)=\left(\begin{array}{c}
-\bar{f}_{1} \\
-\bar{f}_{2} \\
-\bar{f}_{3}
\end{array}\right) ; \quad\left(\begin{array}{ccc}
-\mathcal{P}_{4}^{56} & \mathcal{P}_{2}^{61} & \mathcal{P}_{3}^{15} \\
\mathcal{P}_{1}^{26} & -\mathcal{P}_{5}^{64} & \mathcal{P}_{3}^{42} \\
\mathcal{P}_{1}^{53} & \mathcal{P}_{2}^{34} & -\mathcal{P}_{6}^{45}
\end{array}\right)=\left(\begin{array}{ccc}
\bar{g}_{11} & \bar{g}_{12} & \bar{g}_{13} \\
\bar{g}_{21} & \bar{g}_{22} & \bar{g}_{23} \\
\bar{g}_{31} & \bar{g}_{32} & \bar{g}_{33}
\end{array}\right)
$$

The superpotential generated by the $\mathcal{P}$ fluxes alone is

$$
\begin{aligned}
\mathcal{W}_{\mathcal{P}} & =-S \sum_{i=1}^{3} f_{i} T_{i}+i S \sum_{i, j=1}^{3} U_{j} g_{j i} T_{i}+S U_{2} U_{3} \sum_{i=1}^{3} \bar{g}_{1 i} T_{i}+S U_{1} U_{3} \sum_{i=1}^{3} \bar{g}_{2 i} T_{i} \\
& +S U_{1} U_{2} \sum_{i=1}^{3} \bar{g}_{3 i} T_{i}-i S U_{1} U_{2} U_{3} \sum_{i=1}^{3} \bar{f}_{i} T_{i} .
\end{aligned}
$$


We thus see that we get new superpotential couplings which are linear in $S$ and $T_{i}$ and up to cubic order in the $U_{i}$. The $\mathcal{P}$ fluxes will also give rise to modifications to tadpole conditions and to Jacobi constraints involving these new fluxes. We now discuss these issues in turn.

\subsection{S-dual tadpoles}

The $C_{4}$ tadpole term (2.34) is S-duality invariant because $C_{4}$ is invariant whereas $\overline{\mathcal{F}}_{3}$ and $\overline{\mathcal{H}}_{3}$ transform as in (4.4). On the contrary, the $C_{8}$ tadpole (3.7) is not S-duality invariant, as one can easily check. In fact this is expected from the known fact [27, 28, 29] that the $C_{8}$ RR-form is one component in a $S L(2, \mathbb{Z})$ triplet of 8 -forms, $\left(C_{8}, \widetilde{C}_{8}, C_{8}^{\prime}\right)$. Under $S \rightarrow 1 / S$ they transform as

$$
\begin{aligned}
& C_{8} \longrightarrow-\widetilde{C}_{8}, \\
& \widetilde{C}_{8} \longrightarrow-C_{8}, \\
& C_{8}^{\prime} \longrightarrow-C_{8}^{\prime} .
\end{aligned}
$$

There is a constraint among the field strengths of these 8-forms so that there are only two propagating degrees of freedom. These three forms may be sourced by three types of 7-branes, D7-branes, NS7-branes and certain other 7-branes called I7 in [30]. In our factorized torus each of them will come in three varieties, $\mathrm{D}_{i}, \mathrm{NS}_{i}$ and $\mathrm{I} 7_{i}$, with $i=$ 1,2,3, labelling one of the three tori transverse to the brane. Starting from eq.(3.7) and imposing $S L(2, \mathbb{Z})_{S}$ invariance of the action one arrives at

$$
\int_{M_{4} \times T^{6}}-C_{8} \wedge \mathcal{Q} \overline{\mathcal{F}}_{3}+\widetilde{C}_{8} \wedge \mathcal{P} \overline{\mathcal{H}}_{3}+C_{8}^{\prime} \wedge\left(\mathcal{Q} \overline{\mathcal{H}}_{3}+\mathcal{P} \overline{\mathcal{F}}_{3}\right)
$$

Just like $\mathcal{Q} \overline{\mathcal{F}}_{3}$, here $\mathcal{P} \overline{\mathcal{H}}_{3}, \mathcal{Q} \overline{\mathcal{H}}_{3}$ and $\mathcal{P} \overline{\mathcal{F}}_{3}$ are 2 -forms computed as in (3.2). For example, $(\mathcal{Q} \overline{\mathcal{H}})_{L M}=\frac{1}{2} \mathcal{Q}_{[L}^{A B} \overline{\mathcal{H}}_{M] A B}$.

The first two terms in eq. (4.11) give rise to tadpoles of $\mathrm{D} 7_{i}$-branes and their $\mathrm{S}$-dual $\mathrm{NS}_{i}$-branes. Looking at components we obtain the cancellation conditions

$$
\begin{aligned}
-N_{\mathrm{D} 7_{i}}+\frac{1}{2}\left[m h_{i}-e_{0} \bar{h}_{i}-\sum_{j}\left(q_{j} b_{j i}+e_{j} \bar{b}_{j i}\right)\right] & =0, \\
-N_{\mathrm{NS}_{i}}+\frac{1}{2}\left[h_{0} \bar{f}_{i}-\bar{h}_{0} f_{i}-\sum_{j}\left(\bar{a}_{j} g_{j i}-a_{j} \bar{g}_{j i}\right)\right] & =0 .
\end{aligned}
$$


Concerning the third term, one observes that the flux combinations coupling to $C_{8}^{\prime}$ do not have RR character. In fact they are rather related to NS Bianchi identities. In particular, in section 3.4 we found that $\mathcal{Q}_{[L}^{A B} \overline{\mathcal{H}}_{M N] B}=0$. It is easy to show that for our class of fluxes this then implies $\mathcal{Q} \overline{\mathcal{H}}_{3}=0$. We discuss further this issue in the next section.

\subsection{S-dual Bianchi constraints}

In section 3.4 we discussed the Bianchi identities leading to constraints on the $\overline{\mathcal{H}}_{3}$ and $\mathcal{Q}$ fluxes. By S-duality we expect constraints on the RR fluxes $\overline{\mathcal{F}}_{3}$ and the new fluxes $\mathcal{P}$. To begin we consider the identity (3.29). To achieve closure under $S L(2, \mathbb{Z})_{S}$ we find that the condition $\mathcal{Q}_{P}^{[M N} \mathcal{Q}_{R}^{L] P}=0$, schematically $\mathcal{Q} \cdot \mathcal{Q}=0$, remains valid and that there are actually two new constraints. The point is that $\mathcal{Q} \cdot \mathcal{Q}$ is a component in a triplet of $S L(2, \mathbb{Z})_{S}$. Acting with $S \rightarrow 1 / S$ we find that another component is $\mathcal{P} \cdot \mathcal{P}$, with corresponding Bianchi identity given by

$$
\mathcal{P}_{P}^{[M N} \mathcal{P}_{R}^{L] P}=0 .
$$

Finally, applying a translation $S \rightarrow(S+i)$ shows that the third triplet component is $(\mathcal{Q} \cdot \mathcal{P}+\mathcal{P} \cdot \mathcal{Q})$. Thus, there is also a constraint

$$
\mathcal{Q}_{P}^{[M N} \mathcal{P}_{R}^{L] P}+\mathcal{P}_{P}^{[M N} \mathcal{Q}_{R}^{L] P}=0 .
$$

Notice that the left hand side has net RR charge so that it is potentially related to tadpoles. However, given the tensor structure, it is not clear how it could couple to the known RR forms. In absence of sources we are led to enforce the equality to zero.

In components, eq. (4.14) breaks into $(i \neq j \neq k)$

$$
\begin{aligned}
-g_{i i} g_{j k}+\bar{g}_{k i} f_{k}+f_{i} \bar{g}_{k k}-g_{j i} g_{i k} & =0, \\
-\bar{g}_{i i} \bar{g}_{j k}+g_{k i} \bar{f}_{k}+\bar{f}_{i} g_{k k}-\bar{g}_{j i} \bar{g}_{i k} & =0, \\
-g_{i i} \bar{g}_{i j}+\bar{g}_{j i} g_{j j}+f_{i} \bar{f}_{j}-g_{k i} \bar{g}_{k j} & =0, \\
\bar{g}_{i i} g_{i j}-g_{j i} \bar{g}_{j j}+f_{i} \bar{f}_{j}-g_{k i} \bar{g}_{k j} & =0 .
\end{aligned}
$$

These are completely analogous to the conditions following from $\mathcal{Q}_{P}^{[M N} \mathcal{Q}_{R}^{L] P}=0$, given in eqs. (3.30)-(3.33). In the particular case in which one imposes a symmetry under exchange 
of the three sub-tori one has

$$
\begin{aligned}
& f_{i}=f \quad ; \quad \bar{f}_{i}=\bar{f} ; \\
& g_{i j}=g(i \neq j) \quad ; \quad g_{i i}=\gamma \quad ; \quad \bar{g}_{i j}=\bar{g}(i \neq j) \quad ; \quad \bar{g}_{i i}=\bar{\gamma} .
\end{aligned}
$$

We have further assumed $g_{i j}=g_{j i}$ and $\bar{g}_{i j}=\bar{g}_{j i}$. In this case we find the simplified set of conditions

$$
\begin{aligned}
f(\bar{g}+\bar{\gamma})-g(g+\gamma) & =0 \\
\bar{f}(g+\gamma)-\bar{g}(\bar{g}+\bar{\gamma}) & =0 \\
f \bar{f}-g \bar{g} & =0
\end{aligned}
$$

A simple solution is $f=\bar{f}=g=\bar{g}=0$, but $\gamma, \bar{\gamma} \neq 0$. Another solution is $\gamma=-g$, $\bar{\gamma}=-\bar{g}$ and $\bar{f}=g \bar{g} / f$.

Concerning eq. (4.15), it gives rise to the four additional constraints $(i \neq j \neq k)$

$$
\begin{aligned}
& b_{k k} \bar{g}_{k j}-h_{k} \bar{f}_{j}-\bar{b}_{j k} g_{j j}+b_{i k} \bar{g}_{i j}+g_{k k} \bar{b}_{k j}-f_{k} \bar{h}_{j}-\bar{g}_{j k} b_{j j}+g_{i k} \bar{b}_{i j}=0, \\
& b_{k k} g_{i j}-h_{k} \bar{g}_{j j}-\bar{b}_{j k} f_{j}+b_{i k} g_{k j}+g_{k k} b_{i j}-f_{k} \bar{b}_{j j}-\bar{g}_{j k} h_{j}+g_{i k} b_{k j}=0, \\
& \bar{b}_{k k} \bar{g}_{i j}-\bar{h}_{k} g_{j j}-b_{j k} \bar{f}_{j}+\bar{b}_{i k} \bar{g}_{k j}+\bar{g}_{k k} \bar{b}_{i j}-\bar{f}_{k} b_{j j}-g_{j k} \bar{h}_{j}+\bar{g}_{i k} \bar{b}_{k j}=0, \\
& \bar{b}_{k k} g_{k j}-\bar{h}_{k} f_{j}-b_{j k} \bar{g}_{j j}+\bar{b}_{i k} g_{i j}+\bar{g}_{k k} b_{k j}-\bar{f}_{k} h_{j}-g_{j k} \bar{b}_{j j}+\bar{g}_{i k} b_{i j}=0 .
\end{aligned}
$$

For isotropic fluxes (3.5) and (4.20) they reduce to

$$
\begin{aligned}
b \bar{g}+g \bar{b}-h \bar{f}-f \bar{h} & =0 \\
g(b+\beta)-h(\bar{g}+\bar{\gamma})-f(\bar{b}+\bar{\beta})+b(g+\gamma) & =0 \\
\bar{g}(\bar{b}+\bar{\beta})-\bar{h}(g+\gamma)-\bar{f}(b+\beta)+\bar{b}(\bar{g}+\bar{\gamma}) & =0 .
\end{aligned}
$$

The simple solution $f=\bar{f}=g=\bar{g}=0$ also works, supplemented with $b \gamma=h \bar{\gamma}$ and $\bar{b} \bar{g}=\bar{h} \gamma$.

Let us now discuss the modifications to the identity eq. (3.24). In this case we can construct the fully $S L(2, \mathbb{Z})_{S}$ invariant condition

$$
\mathcal{Q}_{[L}^{R P} \overline{\mathcal{H}}_{M N] P}-\mathcal{P}_{[L}^{R P} \overline{\mathcal{F}}_{M N] P}=0
$$


Substituting the flux components leads to $(i \neq j \neq k)$

$$
\begin{aligned}
& \bar{h}_{0} h_{j}+\bar{a}_{i} b_{i j}+\bar{a}_{j} b_{j j}-a_{k} \bar{b}_{k j}+m f_{j}-q_{i} g_{i j}-q_{j} g_{j j}-e_{k} \bar{g}_{k j}=0, \\
& h_{0} \bar{h}_{j}+a_{i} \bar{b}_{i j}+a_{j} \bar{b}_{j j}-\bar{a}_{k} b_{k j}-e_{0} \bar{f}_{j}-e_{i} \bar{g}_{i j}-e_{j} \bar{g}_{j j}-q_{k} g_{k j}=0, \\
& \bar{h}_{0} b_{k j}+\bar{a}_{i} \bar{b}_{j j}+\bar{a}_{j} \bar{b}_{i j}-a_{k} \bar{h}_{j}+m g_{k j}-q_{i} \bar{g}_{j j}-q_{j} \bar{g}_{i j}-e_{k} \bar{f}_{j}=0, \\
& h_{0} \bar{b}_{k j}+a_{i} b_{j j}+a_{j} b_{i j}-\bar{a}_{k} h_{j}-e_{0} \bar{g}_{k j}-e_{i} g_{j j}-e_{j} g_{i j}-q_{k} f_{j}=0 .
\end{aligned}
$$

With the simple isotropic fluxes these constraints read

$$
\begin{aligned}
& \bar{h}_{0} h+\bar{a}(b+\beta)-a \bar{b}+m f-q(g+\gamma)-e \bar{g}=0, \\
& h_{0} \bar{h}+a(\bar{b}+\bar{\beta})-\bar{a} b-e_{0} \bar{f}-e(\bar{g}+\bar{\gamma})-q g=0, \\
& \bar{h}_{0} b+\bar{a}(\bar{b}+\bar{\beta})-a \bar{h}+m g-q(\bar{g}+\bar{\gamma})-e \bar{f}=0, \\
& h_{0} \bar{b}+a(b+\beta)-\bar{a} h-e_{0} \bar{g}-e(g+\gamma)-q f=0 .
\end{aligned}
$$

We will discuss some solutions in section 5 .

The combination $\left(\mathcal{Q} \cdot \overline{\mathcal{H}}_{3}-\mathcal{P} \cdot \overline{\mathcal{F}}_{3}\right)$ appearing in the identity $(4.31)$ is an $S L(2, \mathbb{Z})_{S}$ singlet. Now, with the doublets $(\mathcal{Q}, \mathcal{P})$ and $\left(\overline{\mathcal{F}}_{3}, \overline{\mathcal{H}}_{3}\right)$ we can also form a triplet which is rather related to tadpoles of RR 8-forms as we saw in the previous section. In particular, concerning the $C_{8}^{\prime}$ flux tadpole in eq. (4.11), we see that in general it does not cancel because neither $\mathcal{Q} \cdot \overline{\mathcal{H}}_{3}$ nor $\mathcal{P} \cdot \overline{\mathcal{F}}_{3}$ has to vanish separately. In fact, starting with the tadpole term of $C_{8}^{\prime}$ we obtain the cancellation condition

$$
N_{\mathrm{I} 7_{i}}+\frac{1}{2}\left[e_{0} \bar{f}_{i}-m f_{i}+\sum_{j}\left(q_{j} g_{j i}+e_{j} \bar{g}_{j i}\right)\right]=0
$$

where we have taken into account the constraints following from (4.31).

Unlike the situation with only NS, RR and geometric fluxes, we do not have at the moment a prescription, e.g. by doing generalized dimensional reduction of a 10-dimensional theory, to obtain the S-dual equivalent of the Bianchi identities. We have thus used as a guide the duality transformations. It would nevertheless be very interesting to have methods different from duality arguments in order to obtain the complete set of constraints. 


\section{$5 \mathcal{N}=1$ Minkowski minima with moduli fixed}

In this section we intend to start analyzing the landscape of vacua of the moduli potential when S-dual fluxes are turned on. The essential new feature in the superpotential will be the presence of terms (in type IIB langauge) of the form $S T_{i} P\left(U_{j}\right)$, with $P\left(U_{j}\right)$ a cubic polynomial with integer flux coefficients. The complete superpotential is the sum of eqs. (3.4) and (4.9). To study generic supersymmetric and non-supersymmetric vacua of the ensuing moduli potential is beyond the scope of this work. As a first step towards exploring the effect of S-dual fluxes we will only look here for supersymmetric Minkowski solutions in which the potential attains a minimum when the superpotential and its partial derivatives vanish.

We will work in IIB/O3 and restrict to the case with $T_{i}=T, U_{i}=U$ and fluxes given by (3.5) and (4.20). The superpotential then simplifies to

$$
\begin{aligned}
\mathcal{W} & =e_{0}+3 i e U-3 q U^{2}+i m U^{3} \\
& +S\left[i h_{0}-3 a U+3 i \bar{a} U^{2}-\bar{h}_{0} U^{3}\right] \\
& +3 T\left[-i h-(2 b+\beta) U+i(2 \bar{b}+\bar{\beta}) U^{2}+\bar{h} U^{3}\right] \\
& +3 S T\left[-f+i(2 g+\gamma) U+(2 \bar{g}+\bar{\gamma}) U^{2}-i \bar{f} U^{3}\right] .
\end{aligned}
$$

In this isotropic case the tadpole cancellation conditions become

$$
\begin{aligned}
N_{\mathrm{D} 3}+\frac{1}{2}\left[m h_{0}-e_{0} \bar{h}_{0}+3 q a+3 e \bar{a}\right] & =16, \\
-N_{\mathrm{D} 7}+\frac{1}{2}\left[m h-e_{0} \bar{h}-q(2 b+\beta)-e(2 \bar{b}+\bar{\beta})\right] & =0 \\
-N_{\mathrm{NS} 7}+\frac{1}{2}\left[h_{0} \bar{f}-\bar{h}_{0} f-\bar{a}(2 g+\gamma)+a(2 \bar{g}+\bar{\gamma})\right] & =0 . \\
N_{\mathrm{I} 7}+\frac{1}{2}\left[e_{0} \bar{f}-m f+q(2 g+\gamma)+e(2 \bar{g}+\bar{\gamma})\right] & =0 .
\end{aligned}
$$

The fluxes must further satisfy the constraints derived in sections 3.4 and 4.2 .

It is helpful to make a change of variables

$$
U=-i \rho \quad ; \quad S=-i \sigma \quad ; \quad T=-i \tau .
$$


The superpotential (5.1) then becomes

$$
\mathcal{W}=E_{1}+\sigma E_{2}+\tau E_{3}+\sigma \tau E_{4}
$$

where the $E_{i}$ are cubic polynomials in $\rho$ given by

$$
\begin{aligned}
& E_{1}=e_{0}+3 e \rho+3 q \rho^{2}-m \rho^{3}, \\
& E_{2}=h_{0}+3 a \rho-3 \bar{a} \rho^{2}-\bar{h}_{0} \rho^{3}, \\
& E_{3}=3\left[-h+(2 b+\beta) \rho-(2 \bar{b}+\bar{\beta}) \rho^{2}+\bar{h} \rho^{3}\right], \\
& E_{4}=3\left[f-(2 g+\gamma) \rho+(2 \bar{g}+\bar{\gamma}) \rho^{2}-\bar{f} \rho^{3}\right] .
\end{aligned}
$$

The advantage is that now all coefficients are real, in fact integers.

The problem is to find solutions of

$$
\mathcal{W}=\frac{\partial \mathcal{W}}{\partial \rho}=\frac{\partial \mathcal{W}}{\partial \sigma}=\frac{\partial \mathcal{W}}{\partial \tau}=0
$$

To begin, let us review the known situation in which there are neither $\mathcal{Q}$ nor $\mathcal{P}$ fluxes [38]. In this case $E_{3}=E_{4}=0$ and $\mathcal{W}$ does not depend on $\tau$. From $\partial \mathcal{W} / \partial \sigma=0$ and $\mathcal{W}=0$ we find $E_{1}=E_{2}=0$. The remaining equation $\partial \mathcal{W} / \partial \rho=0$ gives $\sigma=-E_{1}^{\prime} / E_{2}^{\prime}$ (where prime denotes derivative with respect to $\rho$ ). The task is to determine whether $E_{1}=0$ and $E_{2}=0$ have a common root $\rho=\rho_{0}$ with $\rho_{0}$ necessarily complex (so that $\operatorname{Re} U \neq 0$ at the minimum). Now, $\rho_{0}^{*}$ must also be a root because the $E_{i}$ have real coefficients. Thus, the $E_{i}$ must factorize as [38]

$$
E_{i}=\left(\rho-\rho_{0}\right)\left(\rho-\rho_{0}^{*}\right)\left(\mu_{i} \rho+\nu_{i}\right)
$$

with $\mu_{i}$ and $\nu_{i}$ some coefficients that depend on the fluxes. In [38] it is shown that there are fluxes that allow such a factorization and moreover lead to $\operatorname{Re} S>0$. These fluxes contribute to the $C_{4}$ tadpole as D3-branes.

In a similar spirit we can consider the case $\overline{\mathcal{H}}_{3}=0$ and $\mathcal{P}=0$ in which $E_{2}=E_{4}=0$ and $\mathcal{W}$ does not depend on $\sigma$. Now $E_{1}$ and $E_{3}$ must factorize as in (5.7). For $E_{1}$ this poses no problem because the fluxes $e_{0}, e, q$ and $m$ are unconstrained when $\mathcal{P}=0$. However, for the coefficients of $E_{3}$ we have the Bianchi conditions (3.42). These can be satisfied taking 
for example $h=0, \bar{b}=0$ and $\beta=-b$. To simplify we also take $\bar{\beta}=0$. Then, $\rho_{0}=i \sqrt{b / \bar{h}}$ which requires $\bar{h} b>0$. Imposing that $E_{1}$ has the same root gives the conditions $e_{0} \bar{h}=3 q b$ and $m e_{0}=-9 e q$ which can be satisfied with $m=e=0$. It is also instructive to compute $\tau=-E_{1}^{\prime} / E_{3}^{\prime}$. We find $\tau=q \rho_{0} / b$. Then $\operatorname{Re} T>0$ requires $q b>0$ and this implies that the $C_{8}$ tadpole is negative (same sign as for a D7-brane).

To continue the systematic analysis we can set only the $\mathcal{P}$-fluxes to zero so that $E_{4}=0$. Now minimization with $\mathcal{W}=0$ requires $E_{1}=E_{2}=E_{3}=0$. Moreover, the coefficients of $E_{2}$ and $E_{3}$ must verify Bianchi constraints. Fulfilling the constraints by using the solution (3.43) leads to $E_{3}$ without complex roots. Taking instead the constraint solution (3.44) we find that $E_{3}$ could have a complex root only if $h_{0} \bar{a}>0$, but it cannot be a simultaneous root of $E_{2}$. The interesting conclusion is that to fix all moduli in a supersymmetric Minkowski minimum, within our class of solutions of Bianchi constraints, we have to go beyond metric and non-geometric fluxes.

We now come to the generic situation with all fluxes turned on. From $\partial \mathcal{W} / \partial \sigma=0$ and $\partial \mathcal{W} / \partial \tau=0$ we find

$$
\tau=-\frac{E_{2}}{E_{4}} \quad ; \quad \sigma=-\frac{E_{3}}{E_{4}} .
$$

Substituting in $\mathcal{W}=0$ and $\partial \mathcal{W} / \partial \rho=0$ then gives

$$
E=E_{1} E_{4}-E_{2} E_{3}=0 \quad ; \quad E^{\prime}=0 \text {. }
$$

Thus, this $E$ must have a double root $\rho_{0}$, necessarily complex. We also know that $E$ has real coefficients and is generically of order six in $\rho$. Hence, it can be written as

$$
E=3\left(\rho-\rho_{0}\right)^{2}\left(\rho-\rho_{0}^{*}\right)^{2}\left(\alpha \rho^{2}+\delta \rho+\epsilon\right)
$$

where $\alpha, \delta, \epsilon$ and $\rho_{0}$ depend on the fluxes.

To proceed further we will implement specific solutions for the Bianchi identities derived in section 4.2. We consider different cases according to how we solve the constraints on $\mathcal{P}$ and $\mathcal{Q}$ fluxes alone. These cases are shown in table 4 , where we have also displayed the solution to the constraint involving the $\overline{\mathcal{F}}_{3}$ and $\overline{\mathcal{H}}_{3}$ fluxes.

Within our class of solutions the polynomials $E_{3}$ and $E_{4}$ always take a simpler form. For example, in case 1 they are given by

$$
E_{3}=\frac{3}{h}\left(\bar{b} \rho^{2}+h\right)(b \rho-h) \quad ; \quad E_{4}=\frac{3 \gamma \rho}{h}(b \rho-h) .
$$




\begin{tabular}{|c|c|c|c|c|}
\hline Case & $\mathcal{P} \cdot \mathcal{P}=0$ & $\mathcal{Q} \cdot \mathcal{Q}=0$ & $\mathcal{Q} \cdot \mathcal{P}+\mathcal{P} \cdot \mathcal{Q}=0$ & $\mathcal{Q} \cdot \overline{\mathcal{H}}_{3}-\mathcal{P} \cdot \overline{\mathcal{F}}_{3}=0$ \\
\hline & $f=\bar{f}=g=\bar{g}=0$ & $\beta=-b, \bar{\beta}=-\bar{b}$ & & \\
1 & $h \bar{h}=b \bar{b}$ & $h_{0} \bar{b}=e \gamma+\bar{a} h$ \\
& & & $\bar{h}_{0} h=q \gamma+a \bar{\gamma}$ \\
\hline 2 & $\gamma=-g, \bar{\gamma}=-\bar{g}$ & $h=\bar{h}=b=\bar{b}=0$ & $f \bar{\beta}=g \beta$ & $m g=e \bar{f}-\bar{a} \bar{\beta}$ \\
& $f \bar{f}=g \bar{g}$ & & $q g=a \bar{\beta}-e_{0} \bar{f}$ \\
\hline
\end{tabular}

Table 4: Solutions to Bianchi identities

In each case we compute $E$ and check if it can be factorized in the form (5.10). When this is possible we can determine $\rho_{0}$. Moreover, typically there will be relations among the fluxes. We have limited ourselves to finding some solutions. Details are presented below.

\section{Case 1}

We find a class of minima with fluxes satisfying the relations

$$
q=0 \quad ; \quad e_{0} \gamma=4 a h \quad ; \quad m h_{0} h \gamma=(e \gamma+4 h \bar{a})(e \gamma+h \bar{a})
$$

Besides, $q=0$ implies $h \bar{h}_{0}=a \bar{b}$, with $\bar{b}$ given in table 4 . As free parameters we can then take $a, \bar{a}, b, h_{0}, h, \gamma$ and $e$. They must be such that the remaining dependent fluxes come out integers as well. Furthermore, there are sign relations required for consistency. For example, we find

$$
\rho_{0}=i\left|\rho_{0}\right| \quad ; \quad\left|\rho_{0}\right|^{2}=-\frac{h_{0} h}{e \gamma+h \bar{a}} .
$$

This needs $h_{0} h(e \gamma+h \bar{a})<0$, then $U=\sqrt{-h_{0} h /(e \gamma+h \bar{a})}$.

The remaining moduli turn out to be

$$
\begin{aligned}
S & =\frac{2 h}{\gamma U}, \\
T & =\frac{h\left[h_{0}(2 \bar{a} h-e \gamma)-2 i a U(e \gamma+\bar{a} h)\right]}{3 \gamma U(e \gamma+\bar{a} h)(h-i b U)} .
\end{aligned}
$$

To guarantee $\operatorname{Re} S>0$ and $\operatorname{Re} T>0$ we need

$$
h \gamma>0 \quad ; \quad(2 a b-2 \bar{a} h+e \gamma)>0 .
$$

For example, choosing

$$
a=-8 ; b=-4 ; h=-4 ; \gamma=-4 ; \bar{a}=12 ; h_{0}=8 ; e=-16
$$


we find that all dependent fluxes are also even integers. The moduli are determined to be

$$
U=S=\sqrt{2} \quad ; \quad T=\frac{1}{9}(14 \sqrt{2}+16 i)
$$

It is also interesting to compute the tadpoles. In particular we find

$$
N_{\mathrm{D} 3}-288=16 ;-N_{\mathrm{D} 7}+80=0 ;-N_{\mathrm{NS} 7}+40=0 \quad ; \quad N_{\mathrm{I} 7}+32=0 .
$$

We observe the peculiar result that fluxes contribute to the $C_{4}$ tadpole as O3-planes instead of D3-branes. However, this is not generic. In other examples the $C_{4}$ flux tadpole comes out positive. The 8-form tadpoles can have either sign, or even cancel, depending on the parameters.

Within the above class of vacua we can set $e=0$, implying $h_{0} \bar{b}=\bar{a} h$. The I7 tadpole cancels since now $q=e=0$. On the other hand, from the condition $\operatorname{Re} S>0$ we conclude that in this case fluxes always contribute to the $C_{4}$ tadpole as D3-branes. Using also the condition for $\operatorname{Re} T>0$ shows that the flux piece in the $C_{8}$ tadpole is positive (opposite sign as D7-branes). The flux piece in the $\operatorname{NS} 7\left(\widetilde{C}_{8}\right)$ tadpole is also positive. In fact, the flux tadpoles of $C_{8}$ and $\widetilde{C}_{8}$ are both positive and proportional to Re $T$. To give a numerical example, we can take $\bar{a}=-h_{0}=2, U=1$, and

$$
a=-8 \quad ; \quad b=-2 \quad ; \quad h=-2 \quad ; \quad \gamma=-2 \quad ; \quad S=2 \quad ; \quad T=\frac{5}{3}-i
$$

It is easy to verify that the dependent fluxes are all even integers.

If we relax the condition $b \gamma=h \bar{\gamma}$ we can find vacua with all 7-brane tadpoles zero but $\operatorname{Re} T \neq 0$. To cancel these tadpoles from the beginning we impose $e=q=0$, together with $m h=e_{0} \bar{h}$ and $a \bar{\gamma}=\bar{a} \gamma$. We then find a solution provided that we also fulfill the relations

$$
\frac{h a}{b \bar{a}}>0 \quad ; \quad a^{2}=-h_{0} \bar{a} \quad ; \quad m \gamma=-\frac{\bar{a}\left(a h-b h_{0}\right)^{2}}{h h_{0}^{2}} .
$$

As independent fluxes we can now choose $\bar{a}, b, a, h$ and $\gamma$. For the moduli we obtain

$$
\begin{aligned}
U & =\sqrt{\frac{h a}{b \bar{a}}} \\
S & =\frac{-i b(a+i \bar{a} U)^{2}}{\gamma a^{2}}, \\
T & =\frac{(a-i \bar{a} U)^{2}}{3 \gamma \bar{a} U} .
\end{aligned}
$$


To have $\operatorname{Re} S>0$ it suffices to impose $h \gamma>0$. The flux contribution to the D3 tadpole is then positive. We can also arrange to have all fluxes to be integers while $\operatorname{Re} T$ is positive and large. However, the flux D3 tadpole will also be large.

\section{Case 2}

There is a type of solutions with free parameters $\beta, \bar{\beta}, e_{0}, e, \bar{f}, g$ and $a$, with remaining fluxes determined by

$$
\bar{a}=0 \quad ; \quad h_{0} \bar{\beta}=4 e g \quad ; \quad \bar{h}_{0} \bar{\beta}=\frac{\bar{f}\left(4 e_{0} \bar{f}-3 a \bar{\beta}\right)}{g} .
$$

Notice that then $m=e \bar{f} / g$ and $q$ is given in table 4. There is a sign condition $g \bar{f}<0$, then $U=\sqrt{-g / \bar{f}}$. We further obtain

$$
\begin{aligned}
S & =\frac{\bar{\beta}}{2 \bar{f} U}, \\
T & =\frac{U}{3 g} \frac{\left[2 e \bar{f} U+i\left(2 e_{0} \bar{f}-3 a \bar{\beta}\right)\right]}{(\bar{\beta} U+i \beta)} .
\end{aligned}
$$

It is easy to check that $\operatorname{Re} S>0$ and $\operatorname{Re} T>0$ are positive as long as

$$
g \bar{\beta}<0 \quad ; \quad\left(2 e g+3 a \beta-\frac{2 e_{0} \beta \bar{f}}{\bar{\beta}}\right)>0 .
$$

For an illustrative example, consider the parameters

$$
\bar{f}=2 ; \beta=8 ; \bar{\beta}=8 ; g=-2 ; a=28 ; e_{0}=16 ; e=96
$$

The moduli are then fixed as

$$
U=1 \quad ; \quad S=2 \quad ; \quad T=\frac{1}{3}(7+31 i)
$$

For the flux-induced tadpoles we obtain

$$
N_{\mathrm{D} 3}+32=16 \quad ; \quad N_{\mathrm{I} 7}+112=0 .
$$

The flux contribution to $C_{8}$ and $\widetilde{C}_{8}$ tadpoles is zero.

The I7 tadpoles cancel when $a=0$. Then it is simpler to show that the free parameters can be chosen so that all other fluxes are integers while $\operatorname{Re} S$ and $\operatorname{Re} T$ are large and 
positive. The sign relations among the parameters imply that the $C_{4}, C_{8}$ and $\widetilde{C}_{8}$ flux tadpoles are positive. The latter two are proportional to $\operatorname{Re} T$.

Similar to case 1 , if we relax the condition $f \bar{\beta}=g \beta$ we can find minima with all 7-brane tadpoles zero but $\operatorname{Re} T \neq 0$.

Other solutions of the Bianchi identities can be obtained by combining the building blocks of table 4 . For example, $\mathcal{P} \cdot \mathcal{P}=0$ can be fulfilled as in case 2 , and $\mathcal{Q} \cdot \mathcal{Q}=0$ as in case 1 . Then the solution of $\mathcal{Q} \cdot \mathcal{P}+\mathcal{P} \cdot \mathcal{Q}=0$ can be written as $(b f-g h)(h \bar{f}-g \bar{b})=0$. Now, if $h \bar{f}=g \bar{b}, E_{3}$ and $E_{4}$ have a common quadratic factor and it can then be shown that the polynomial $E$ cannot be factorized as needed. When $b f=g h$, to avoid $\operatorname{Re} S=0$ it must be that $U$ is necessarily complex. In this more complicated case we have not been able to find supersymmetric Minkowski minima.

In summary, some differences compared to the type IIB results in ref.[38] without nongeometric nor S-dual fluxes are evident. The situation now is rather more involved but still we have found some concrete results. For simplicity we have analyzed the case with isotropic fluxes and moduli $T_{i}=T, U_{i}=U$. We find Minkowski $\mathcal{N}=1$ vacua in which not only the dilaton and complex structure fields are fixed but also the Kähler modulus $T$ is fixed. However, if we analyze the more general case with independent $T_{i}, U_{i}$ fields, generically only one linear combination of the Kähler moduli $T_{i}$ is fixed. This is due to the fact that the superpotential is only linear in the $T_{i}$ and essentially only depends on a linear combination of these moduli.

When S-dual backgrounds are switched on, the contribution from fluxes to the tadpole of the $\mathrm{RR} C_{4}$ form can have either sign depending on the flux values. This is a surprising result. We know that in absence of S-dual fluxes the $C_{4}$ tadpole due to $\overline{\mathcal{H}}_{3}$ and $\overline{\mathcal{F}}_{3}$ fluxes consistent with the imaginary self-dual condition needed for supersymmetry is always positive $[37,38]$. Concerning the $C_{8}$ tadpole, if only non-geometric fluxes are present, as in a toy example with $\mathcal{W}$ depending only on $U$ and $T$, the flux tadpole is negative (same sign as D7-branes). However, in presence of S-dual backgrounds the flux contribution can be positive (same sign as O7-planes), negative, or even vanish. An analogous result occurs in AdS type IIA vacua with metric fluxes in [8]. The fact that fluxes may contribute to tadpoles as orientifold planes may be useful for model-building as already emphasized in 
$[8]$.

The value of the real parts of the dilaton $S$ and the size modulus $T$ may be made large by appropriately choosing the fluxes. This is in general required to maintain perturbative values for the couplings and the validity of the supergravity approximation. On the other hand, in our supersymmetric Minkowski vacua $\operatorname{Re} S$ and $\operatorname{Re} T$ cannot be made arbitrarily large because in general they are tied to RR tadpoles induced by the fluxes. This could be avoided in AdS vacua, as occurs in the IIA/O6 models without [7] and with metric fluxes [8]. We have not found non-trivial supersymmetric Minkowski vacua with full cancellation of RR tadpoles, although in some cases one can find flux combinations with vanishing contribution to some tadpoles. We assume that localized sources of different kinds may be added to the theory rendering it tadpole free, as it happened in the simpler examples in ref [8]. In this connection, notice that if we want to add D3 and/or D7-branes to vacua like these, the existence of undetermined Kähler $T_{i}$ moduli may in fact be necessary, as emphasized elsewhere [8]. In particular, in the worldvolume of generic branes (not on top of orientifold planes) live $U(1)$ groups that may have triangle anomalies in four dimensions. The $U(1)$ gauge vectors become massive through a generalized Green-Schwarz mechanism by swallowing some linear combination of the $T_{i}$. Thus, for this mechanism to work, some $T_{i}$ fields should be left unfixed by the fluxes. This turns out to be related to the requirement of absence of Freed-Witten brane worldvolume anomalies [8].

\section{Generalized Duality Invariant Superpotentials}

In previous sections we have described fluxes present in different T-dual type II orientifolds. The closed string sector of all these theories, before the addition of fluxes, gives rise at low-energies to an effective $\mathcal{N}=4$ supergravity theory (or $\mathcal{N}=1$ if we further perform a $\mathbb{Z}_{2} \times \mathbb{Z}_{2}$ twist). We would like to compare now to the results obtained from other string constructions having an analogous low-energy structure. Specifically, we would like to compare to the flux-induced superpotential in analogous heterotic compactifications as well as in certain compactifications of M-theory on simple twisted 7-tori [39]. 


\subsection{M-theory on a twisted 7-tori}

We consider here the $G_{2}$-holonomy manifolds $X_{7}$ obtained as certain $\mathbb{Z}_{2} \times \mathbb{Z}_{2} \times \mathbb{Z}_{2}$ orbifolds of the 7-torus, $X_{7}=\mathrm{T}^{7} / \mathbb{Z}_{2} \times \mathbb{Z}_{2} \times \mathbb{Z}_{2}[40]$. We will follow the results and notation used in ref.[39]. One has seven complex moduli fields $M_{I}(x), I=1, \ldots, 7$. They may be defined in terms of the complexified $G_{2}$-form

$$
C_{3}+i \Phi_{3}=i M_{I}(x) \phi^{I}(y)
$$

where $\phi^{I} \in H^{3}\left(X_{7}\right), C_{3}$ is the M-theory 3 -form and $\boldsymbol{\Phi}_{3}=\operatorname{Re} M_{I}(x) \phi^{I}(y)$, with $\operatorname{Re} M_{I}(x)$ parameterizing the volume of the 7 invariant 3-cycles in $X_{7}=T^{7} / \mathbb{Z}_{2} \times \mathbb{Z}_{2} \times \mathbb{Z}_{2}$. We will now consider the addition of metric fluxes in this toroidal model. This is a Scherk-Schwarz reduction which proceeds in a way analogous to that described for type IIA orientifold compactifications. In particular we replace the differentials $d y^{P}, P=1, \cdots, 7$, by twisted forms $\eta^{P}$ satisfying

$$
d \eta^{P}=-\frac{1}{2} \omega_{M N}^{P} \eta^{M} \wedge \eta^{N}, \omega_{[M N}^{P} \omega_{R] P}^{S}=0
$$

where one also has $\omega_{P N}^{P}=0[11,12]$. Among these metric fluxes $\omega_{M N}^{P}$, only twentyone are invariant under the twists. In addition we consider the presence of seven 4 -form backgrounds $g_{I J K L}$ corresponding to fluxes of the M-theory 3-form. The presence of these two types of fluxes gives rise to a superpotential $[41,42,39]$

$$
W_{7}=\frac{1}{4} \int_{X_{7}}(C+i \boldsymbol{\Phi}) \wedge\left[g+\frac{1}{2} d(C+i \boldsymbol{\Phi})\right]+\frac{1}{4} \int_{X_{7}} G_{7}
$$

Here $G_{7}$ is the flux of the 3 -form dual. Expanding this superpotential in terms of the seven moduli in type IIA notation [39] one obtains:

$$
\begin{aligned}
W_{7} & =g_{567891011}+i\left(g_{78910} T_{1}+g_{56910} T_{2}+g_{5678} T_{3}\right)+ \\
& +i\left(g_{57911} S-g_{581011} U_{1}-g_{671011} U_{2}-g_{68911} U_{3}\right) \\
& +\left(\omega_{910}^{11} T_{1} T_{2}+\omega_{56}^{11} T_{2} T_{3}+\omega_{78}^{11} T_{1} T_{3}\right)-S\left(\omega_{79}^{6} T_{1}+\omega_{95}^{8} T_{2}+\omega_{57}^{10} T_{3}\right) \\
& +\left(\omega_{810}^{6} T_{1} U_{1}+\omega_{106}^{8} T_{2} U_{2}+\omega_{68}^{10} T_{3} U_{3}\right)-\left(\omega_{710}^{5} T_{1} U_{2}+\omega_{89}^{5} T_{1} U_{3}+\omega_{105}^{7} T_{2} U_{1}\right) \\
& +\left(\omega_{96}^{7} T_{2} U_{3}+\omega_{58}^{9} T_{3} U_{1}+\omega_{67}^{9} T_{3} U_{2}\right) \\
& -S\left(\omega_{511}^{6} U_{1}+\omega_{711}^{8} U_{2}+\omega_{911}^{10} U_{3}\right)+\omega_{1011}^{9} U_{1} U_{2}+\omega_{611}^{5} U_{2} U_{3}+\omega_{811}^{7} U_{1} U_{3}
\end{aligned}
$$


All terms in this superpotential, except for those in the last line, may be understood in terms of ordinary RR and NS backgrounds in the type IIA orientifold supplemented by metric fluxes. Indeed, all those terms correspond to the fluxes $e_{0}, e_{i}, h_{0}, h_{i}, q_{i}, a_{i}$, and $b_{i j}$, described in chapter 2 . The absence of a $T_{1} T_{2} T_{3}$ term (type IIA mass parameter $m$ ) is expected since in the M-theory scheme considered massive IIA supergravity does not arise.

The new terms appearing in the last line are interesting. The first three correspond to the S-dual fluxes $f_{i}$ introduced before in order to maintain S-duality in the IIB orientifold version of this model. Thus one has the interesting result that the $f_{i}$ fluxes introduced before may be understood as certain ordinary metric fluxes

$$
f_{i}=\omega_{K 11}^{K+1} ; K=5,7,9
$$

in an M-theory version of the same model. On the other hand the last three terms, bilinear in the $U_{i}\left(T_{i}\right)$ in the IIA (IIB) version, are new and are absent even in the extended set of flux-induced superpotential terms discussed in previous chapters. This suggests that there is an even bigger set of flux degrees of freedom to be considered. We will see now that the presence of new terms bilinear and cubic in the $U_{i}$ 's are also expected if we consider fluxes in the heterotic version of the same class of models.

\subsection{Heterotic fluxes}

The type IIA orientifold with O6-planes is T-dual to the type IIB orientifold with O9-

planes, i.e. type I string theory. On the other hand we know that type I is related by S-duality to the $S O(32)$ heterotic string. Therefore, it is interesting to compare the induced superpotentials in both theories. Flux-induced heterotic superpotentials have been analyzed in $[43,44,45,46]$. It has been argued that heterotic H-flux forces the internal manifold $X_{6}$ to be non-Kähler with $d J \neq 0$. Both effects produce a superpotential

$$
W_{\text {het }}=\int_{X_{6}} \Omega \wedge\left(\bar{H}_{\text {het }}+d J_{c}\right)
$$

An example of non-Kähler manifold is the twisted torus in which $d J_{c}=\omega J_{c}$, where $\omega$ are the metric fluxes. 
It is interesting to evaluate $W_{\text {het }}$ in the case of compactification on a factorized $\mathrm{T}^{6}$ with arbitrary metric fluxes on top. The $H$-flux is a generic 3 -form, namely

$$
\bar{H}_{\mathrm{het}}=-e_{0} \alpha_{0}+m \beta_{0}-\sum_{i=1}^{3}\left(q_{i} \alpha_{i}+e_{i} \beta_{i}\right) \text {. }
$$

Our choice of parameters is dictated by the fact that by S-duality $\bar{H}_{\text {het }}$ is equal to the RR flux, given in table 1, of IIB with O9-planes, alias type I. Moreover, the heterotic metric fluxes are the same as those as in IIB/O9 shown in table 2. This means $\omega_{\text {het }}=\boldsymbol{\omega}$. We also need to use that in the toroidal compactification the heterotic complex structure moduli coincide with the geometric parameters, i.e. $U_{i}=\tau_{i}$. The Kähler moduli arise from $J_{c}=i \sum_{j} T_{j} \omega_{j}$. Putting all pieces together we find

$$
\begin{aligned}
W_{\text {het }} & =m+i \sum_{i=1}^{3} q_{i} U_{i}+e_{1} U_{2} U_{3}+e_{2} U_{1} U_{3}+e_{3} U_{1} U_{2}-i e_{0} U_{1} U_{2} U_{3} \\
& +\sum_{i=1}^{3} T_{i}\left[-i \bar{h}_{i}+\sum_{j=1}^{3} \bar{b}_{j i} U_{j}+i b_{1 i} U_{2} U_{3}+i b_{2 i} U_{1} U_{3}+i b_{3 i} U_{1} U_{2}-h_{i} U_{1} U_{2} U_{3}\right] .
\end{aligned}
$$

Superpotentials of this kind have been recently considered in [47]. With isotropic choice of fluxes $W_{\text {het }}$ agrees with results of [5].

Comparing with $(3.17)$ shows that $W_{\text {het }}$ matches $W_{\text {O9 }}$ except for the terms linear in $S$ that are due to non-geometric fluxes $\boldsymbol{R}$ in IIB/O9. Additional $S$-dependent terms in $W_{\text {O9 }}$ will appear if S-dual fluxes are included (T-dual to the $\mathcal{P}$ ). Thus, we conjecture that analogous dilaton-dependent superpotential terms will emerge in the heterotic side from new flux degrees of freedom $R_{\text {het }}$ and $P_{\text {het }}$.

\subsection{Fluxes and $S L(2, \mathbb{Z})^{7}$ duality invariance}

We have just argued that dilaton-dependent terms in $W_{\text {het }}$ would arise from heterotic fluxes $R_{\text {het }}$ and $P_{\text {het }}$. Now, there are reasons to believe that this is not the whole story. In particular, we know that 4-dimensional compactified heterotic strings are self-T-duality invariant. As a consequence, the complete Kähler function, $\mathcal{G}=K+\log |\mathcal{W}|^{2}$, should be invariant under the $S L(2, \mathbb{Z})^{3}$ heterotic T-duality symmetries [48]. In order to be so, the superpotential $W_{\text {het }}$ should transform appropriately. It is easy to convince oneself that 
this demands terms quadratic and cubic in the Kähler moduli $T_{i}$ in $W_{\text {het }}{ }^{2}$. We already observed in section 6.1 that such new quadratic terms seem to be present. We now see that they are also required to help in restoring heterotic self-T-duality.

\begin{tabular}{|c|c|c|c|c|}
\hline \multirow{2}{*}{ Flux } & \multicolumn{4}{|c|}{ components } \\
& \multicolumn{4}{|c|}{ induced terms } \\
\hline$\overline{\mathcal{F}}_{3}$ & $m$ & $q_{i}$ & $e_{i}$ & $e_{0}$ \\
& $U^{3}$ & $U^{2}$ & $U$ & 1 \\
\hline$\overline{\mathcal{H}}_{3}$ & $\bar{h}_{0}$ & $\bar{a}_{i}$ & $a_{i}$ & $h_{0}$ \\
& $S U^{3}$ & $S U^{2}$ & $S U$ & $S$ \\
\hline \multirow{2}{*}{$\mathcal{Q}$} & $\bar{h}_{i}$ & $\bar{b}_{i j}$ & $b_{i j}$ & $h_{i}$ \\
& $T U^{3}$ & $T U^{2}$ & $T U$ & $T$ \\
\hline $\mathcal{P}$ & $\bar{f}_{i}$ & $\bar{g}_{i j}$ & $g_{i j}$ & $f_{i}$ \\
& $S T U^{3}$ & $S T U^{2}$ & $S T U$ & $S T$ \\
\hline \hline$\overline{\mathcal{F}}_{3}^{\prime}$ & $m^{\prime}$ & $q_{i}^{\prime}$ & $e_{i}^{\prime}$ & $e_{0}^{\prime}$ \\
& $T^{3} U^{3}$ & $T^{3} U^{2}$ & $T^{3} U$ & $T^{3}$ \\
\hline$\overline{\mathcal{H}}_{3}^{\prime}$ & $\bar{h}_{0}^{\prime}$ & $\bar{a}_{i}^{\prime}$ & $a_{i}^{\prime}$ & $h_{0}^{\prime}$ \\
& $S T^{3} U^{3}$ & $S T^{3} U^{2}$ & $S T^{3} U$ & $S T^{3}$ \\
\hline $\mathcal{Q}^{\prime}$ & $\bar{h}_{i}^{\prime}$ & $\bar{b}_{i j}^{\prime}$ & $b_{i j}^{\prime}$ & $h_{i}^{\prime}$ \\
& $T^{2} U^{3}$ & $T^{2} U^{2}$ & $T^{2} U$ & $T^{2}$ \\
\hline $\mathcal{P}^{\prime}$ & $\bar{f}_{i}^{\prime}$ & $\bar{g}_{i j}^{\prime}$ & $g_{i j}^{\prime}$ & $f_{i}^{\prime}$ \\
& $S T^{2} U^{3}$ & $S T^{2} U^{2}$ & $S T^{2} U$ & $S T^{2}$ \\
\hline
\end{tabular}

Table 5: IIB/O3 fluxes and their induced terms

To elaborate the point, it helps to look at table 5. The upper half shows the fluxes that we have already encountered in IIB/O3, together with the characteristic term that they induce. $W_{\text {het }}$ contains the monomials due to fluxes of type $\overline{\mathcal{F}}_{3}$ and $\mathcal{Q}$ in the table (upon $h_{i} \leftrightarrow \bar{h}_{i}, e_{0} \leftrightarrow m$, etc.). In order to realize heterotic self T-duality new fluxes of type $\overline{\mathcal{F}}_{3}^{\prime}$ and $\mathcal{Q}^{\prime}$ need to be added. Note that terms quadratic in $T_{i}$ 's, already manifest in the M-theory analysis, come from the flux $h_{i}^{\prime}$. Similarly, self-T-duality of $S$-dependent terms, due to $\overline{\mathcal{H}}_{3}$ and $\mathcal{P}$ in IIB/O3, requires new fluxes $\overline{\mathcal{H}}_{3}^{\prime}$ and $\mathcal{P}^{\prime}$.

All these duality connections among fluxes in different dual incarnations of the same

\footnotetext{
${ }^{2}$ Presence of such terms has also been recently pointed out in [49].
} 
theory suggest that the complete underlying theory is invariant under the $S L(2, \mathbb{Z})^{7}$ transformations corresponding to the seven untwisted moduli in this model. The general flux superpotential will then be a polynomial of degree up to seven on the moduli $M_{I}=\left(S, T_{1}, T_{2}, T_{3}, U_{1}, U_{2}, U_{3}\right)$ and at most linear on any of them. One can write this superpotential in the form:

$$
W_{\text {Flux }}=\sum_{n=0}^{7} D_{i_{1} \ldots i_{n}}^{(n)} M_{i_{1} \ldots M_{n}}
$$

where the $D^{(n)}$ are integer coefficients associated to generalized fluxes ${ }^{3}$. Under $S L(2, \mathbb{Z})_{X}$ the modulus $M_{X}$ transforms as

$$
M_{X} \rightarrow \frac{\left(k_{X} M_{X}-i \ell_{X} M_{X}\right)}{\left(i m_{X} M_{X}+n_{X}\right)} \quad ; \quad k_{X} n_{X}-\ell_{X} m_{X}=1 \quad ; \quad k_{X}, \ell_{X}, m_{X}, n_{X} \in \mathbb{Z} .
$$

The toroidal Kähler potential transforms like

$$
K \rightarrow K+\log \left|i m_{X} M_{X}+n_{X}\right|^{2}
$$

and the complete Kähler function is invariant as long as the fluxes $D^{(n)}$ transform like

$$
\left(D_{i j k . .}^{(n)}, D_{x i j k . .}^{(n+1)}\right) \longrightarrow\left(D_{i j k . .}^{(n)}, D_{x i j k . .}^{(n+1)}\right)\left(\begin{array}{cc}
n_{X} & m_{X} \\
\ell_{X} & k_{X}
\end{array}\right)
$$

The fluxes $D^{(n)}$ may be viewed as symmetric tensors of $n$ indices, with all diagonal components vanishing, thus with binomial coefficient $\left(\begin{array}{l}7 \\ n\end{array}\right)$ independent components. Hence, the total number of generalized fluxes is $\sum_{n=0}^{7}\left(\begin{array}{l}7 \\ n\end{array}\right)=2^{7}=2^{\left(h_{21}+h_{11}+1\right)}$. They provide the 128 components of a representation $(2,2,2,2,2,2,2)$ under $S L(2, \mathbb{Z})^{7}$. As explained in the appendix, this in turn may be embedded into the spinorial 128 of $S O(7,7 ; \mathbb{Z})$. One can decompose the two Weyl spinors of fluxes accordingly to its $S U(7)$ tensorial structure

$$
\begin{aligned}
64 & =1 \oplus 7 \oplus 21 \oplus 35 \\
64^{\prime} & =1^{\prime} \oplus 7^{\prime} \oplus 21^{\prime} \oplus 35^{\prime}
\end{aligned}
$$

The components of each representation are then given by

\footnotetext{
${ }^{3}$ General superpotentials of this type were considered previously in [5] from the point of view of gauged $\mathcal{N}=4$ supergravity.
} 


\begin{tabular}{c|ccccccc} 
Rep. & \multicolumn{1}{|c}{ Flux Components } \\
\hline $\mathbf{1}$ & \multicolumn{1}{c}{$e_{0}$} & & & \\
$\mathbf{7}^{\prime}$ & & & $e_{i}$ & $h_{0}$ & $h_{i}$ & & \\
$\mathbf{2 1}$ & & $q_{i}$ & $a_{i}$ & $b_{i j}$ & $f_{i}$ & $h_{i}^{\prime}$ & \\
$\mathbf{3 5}$ & $m$ & $\bar{a}_{i}$ & $\bar{b}_{i j}$ & $g_{i j}$ & $b_{i j}^{\prime}$ & $f_{i}^{\prime}$ & $e_{0}^{\prime}$ \\
$\mathbf{3 5}$ & $\bar{h}_{0}$ & $\bar{h}_{i}$ & $\bar{g}_{i j}$ & $\bar{b}_{i j}^{\prime}$ & $g_{i j}^{\prime}$ & $e_{i}^{\prime}$ & $h_{0}^{\prime}$ \\
$\mathbf{2 1}$ & & $\bar{f}_{i}$ & $\bar{h}_{i}^{\prime}$ & $\bar{g}_{i j}^{\prime}$ & $q_{i}^{\prime}$ & $a_{i}^{\prime}$ & \\
$\mathbf{7}$ & & & $\bar{f}_{i}^{\prime}$ & $m^{\prime}$ & $\bar{a}_{i}^{\prime}$ & & \\
$\mathbf{1}^{\prime}$ & & & & $\bar{h}_{0}^{\prime}$ & & &
\end{tabular}

Note that in the M-theory setting described above, only the representations $\mathbf{1}, \mathbf{7}^{\prime}$ and $\mathbf{2 1}$ appear explicitly [39].

In terms of component fluxes the full duality covariant superpotential may be written as

$$
\begin{aligned}
W_{\text {Flux }} & =e_{0}-i \sum_{i=1}^{3} h_{i} T_{i}+\frac{1}{2} \sum_{l \neq m \neq n} h_{l}^{\prime} T_{m} T_{n}+i e_{0}^{\prime} T_{1} T_{2} T_{3} \\
& +\left(i h_{0}-\sum_{i=1}^{3} f_{i} T_{i}-\frac{i}{2} \sum_{l \neq m \neq n} f_{l}^{\prime} T_{m} T_{n}-h_{0}^{\prime} T_{1} T_{2} T_{3}\right) S \\
& +\sum_{i=1}^{3}\left[\left(-a_{i}+i \sum_{j=1}^{3} g_{i j} T_{j}-\frac{1}{2} \sum_{l \neq m \neq n} g_{i l}^{\prime} T_{m} T_{n}+i a_{i}^{\prime} T_{1} T_{2} T_{3}\right) S\right. \\
& \left.+i e_{i}-\sum_{j=1}^{3} b_{i j} T_{j}-\frac{i}{2} \sum_{l \neq m \neq n} b_{i l}^{\prime} T_{m} T_{n}-e_{i}^{\prime} T_{1} T_{2} T_{3}\right] U_{i} \\
& +\frac{1}{2} \sum_{r \neq s \neq t}\left[\left(i \bar{a}_{r}+\sum_{j=1}^{3} \bar{g}_{r j} T_{j}+\frac{i}{2} \sum_{l \neq m \neq n} \bar{g}_{r l}^{\prime} T_{m} T_{n}-\bar{a}_{r}^{\prime} T_{1} T_{2} T_{3}\right) S\right. \\
& \left.-q_{r}+i \sum_{j=1}^{3} \bar{b}_{r j} T_{j}-\frac{1}{2} \sum_{l \neq m \neq n} \bar{b}_{r l}^{\prime} T_{m} T_{n}+i q_{r}^{\prime} T_{1} T_{2} T_{3}\right] U_{s} U_{t} \\
& +\left[-\left(\bar{h}_{0}+i \sum_{j=1}^{3} \bar{f}_{j} T_{j}-\frac{1}{2} \sum_{l \neq m \neq n} \bar{f}_{l}^{\prime} T_{m} T_{n}+i \bar{h}_{0}^{\prime} T_{1} T_{2} T_{3}\right) S\right. \\
& \left.+i m+\sum_{j=1}^{3} \bar{h}_{j} T_{j}+\frac{i}{2} \sum_{l \neq m \neq n} \bar{h}_{l}^{\prime} T_{m} T_{n}-m^{\prime} T_{1} T_{2} T_{3}\right] U_{1} U_{2} U_{3}
\end{aligned}
$$

The complexity of this superpotential makes its analysis difficult, except in particular cases like those we have discussed in previous chapters. In any event it is clear that there are 
many parameters which should allow for new possibilities in fixing moduli. It is important to remark that these 128 flux degrees of freedom are not independent. We already saw how Bianchi identities and RR tadpoles strongly restrict the possible fluxes in the simpler case with 64 degrees of freedom. In the most general case analogous constraints should be fulfilled. It would be interesting to have close expressions for these constraints in the more general case.

Note that the above discussion does not imply that the effective action has full $S L(2, \mathbb{Z})^{7}$ duality invariance. Indeed, generic fluxes break these symmetries. Rather, the above discussion shows how the presence of each particular flux explicitly breaks the duality symmetries. As we have seen, some of these flux degrees of freedom have a simple interpretation as metric fluxes or explicit RR or NS backgrounds in some particular version (type IIA or IIB orientifolds, heterotic, M-theory orbifold, ...) of compactified string theory. Some other fluxes do not admit a simple geometric interpretation and yet others are implied by type IIB S-duality and/or heterotic self-T-dualities. Yet all of the 128 fluxes may in general be present in the complete underlying theory.

\section{Final comments and conclussions}

One of the main purposes of this work has been to study the duality properties of the flux degrees of freedom in type II $D=4$ orientifolds, as well as in other related string vacua. The addition of non-geometric fluxes restores T-duality between IIB and IIA theories but spoils type IIB S-duality. We have seen how including new S-dual degrees of freedom this symmetry may be recovered. Once that is done, extra moduli dependent terms appear in the effective superpotentials. Taking into account these new terms we were able to find type IIB Minkowski $\mathcal{N}=1$ vacua in which not only dilaton and complex structure but also some Kähler moduli are fixed. In these classes of vacua we find that fluxes may contribute to RR tadpoles with the same or opposite sign to that of D3- and D7-branes, depending on the flux choice. This fact was already found for type IIA AdS vacua in [8] and may be relevant also for model-building. We leave a more systematic analysis and search for other minima for future work. 
The new and old fluxes are subject to a number of Bianchi and RR tadpole cancellation conditions. We made use of S-duality $S L(2, \mathbb{Z})$ transformations to deduce the form of the new conditions involving all these fluxes. It would be clearly interesting to derive those conditions from other arguments independent from dualities. It would also be important to understand the structure of branes which may be added in these generalized backgrounds and possible constraints which they may suffer. It is known that fluxes may give rise to anomalies in the world-volume of branes and similar effects are expected in the presence of new generalized fluxes.

Dualities relating these theories to heterotic and M-theory compactifications suggest the existence of yet further flux degrees of freedom, giving rise to yet more terms in the effective superpotential. In our toroidal examples a fully $S L(2, \mathbb{Z})^{7}$ covariant superpotential implies the existence of $2^{7}$ fluxes. The general superpotential contains all possible monomials of the seven moduli which are at most linear in any of them, with integer coefficients given by the $2^{7}$ fluxes. Many points remain to be better understood. It would be important to examine the origin and structure of the novel S-dual fluxes as well as ways to understand the generalized constraints on fluxes. The same applies to the extra flux degrees of freedom which might be required to get consistency with the full underlying duality symmetries.

Although we have concentrated on a particular class of toroidal orientifolds we believe that many of the points discussed (like e.g. the explicit expressions of superpotentials in terms of integrals of fluxes over the compact space) should have a more general validity. What we find seems to indicate the existence of a large number of flux degrees of freedom $\left(2^{\left(1+h_{21}+h_{11}\right)}\right.$ in our examples) giving rise to a very rich superpotential in which most or perhaps all moduli might be fixed.

\section{Acknowledgments}

We thank E. Andrés, F. Marchesano, T. Ortín, S. Theisen, and especially A. Uranga for useful discussions. G.A. thanks the Departamento de Física Teórica, Universidad Autónoma de Madrid, for hospitality. G.A. work is partially supported by ANPCyT grant PICT11064. The work of P.G.C. is supported by the Ministerio de Educación y Ciencia 
(Spain) through a FPU grant. P.G.C. thanks the Perimeter Institute for hospitality while working on this paper. A.F. thanks the Max-Planck-Institut für Gravitationsphysik for hospitality while preparing this work. This work has been partially supported by the European Commission under the RTN European Program MRTN-CT-2004-503369 and the CICYT (Spain). 


\section{A. Spinorial embedding of background fluxes.}

We have seen in section 6 how the generalized duality invariant superpotential presents a $S L(2, \mathbb{Z})^{7}$ symmetry. In this appendix we describe in detail how the fluxes are arranged into this structure and their embedding into the spinorial representation of $S O(7,7 ; \mathbb{Z})$.

Each of the seven $S L(2, \mathbb{Z})_{X}$ factors consists of two generators

$$
\mathcal{S}_{X, 1}=\left(\begin{array}{cc}
1 & 1 \\
0 & 1
\end{array}\right) \quad ; \quad \mathcal{S}_{X, 2}=\left(\begin{array}{cc}
0 & -1 \\
1 & 0
\end{array}\right)
$$

acting on the modulus $M_{X}$. From equation (6.10) one can see that $\mathcal{S}_{X, 1}$ corresponds to shifts on the corresponding axion and $\mathcal{S}_{X, 2}$ to M-duality $M_{X} \rightarrow 1 / M_{X}$.

The set of fluxes, denoted $\mathbb{G}$, contains 128 weights of the form $( \pm, \pm, \pm, \pm, \pm, \pm, \pm)$, where \pm stands for $\pm \frac{1}{2}$. The transformation $M_{X} \rightarrow 1 / M_{X}$ is simply given by

$$
\mathcal{S}_{X, 2}\left(n_{1}, \ldots, n_{X}, \ldots, n_{7}\right)=\operatorname{Sign}\left(n_{X}\right)\left(n_{1}, \ldots,-n_{X}, \ldots, n_{7}\right)
$$

Thus, eq.(6.14) transforms in such a way that the full supergravity scalar potential is invariant under the $\mathcal{S}_{X, 2}$ generators. The resulting map between weights and flux components is presented in table 6 . We see, for instance, that $\overline{\mathcal{F}}_{3}$ fluxes (table 1 ) correspond to $\left(+,+,+,+, \underline{\underline{ \pm}, \pm, \pm}\right.$ ) while $\overline{\mathcal{H}}_{3}$ fluxes (table 3$)$ are represented by $(-,+,+,+, \pm, \pm, \pm$ ) spinorial weights.

¿From table 6 we can easily read the action of the duality group in the different fluxes. Notice also that the duality transformations can be easily obtained by expressing the $S L(2, \mathbb{Z})$ generators in terms of lowering and raising operators. Namely, $\mathcal{S}_{X, 2}=$ $\mathcal{S}_{X,+}-\mathcal{S}_{X,-}$ and $\mathcal{S}_{X, 1}=\mathcal{I}+\mathcal{S}_{X,-}$. Thus, for instance, $\mathcal{S}_{1,2} \overline{\mathcal{F}}_{3}=-\overline{\mathcal{H}}_{3}$, corresponds to the S-duality transformation (4.4).

It is interesting to note how half of the degrees of freedom of each of the two Weyl spinors on which $\mathbb{G}=\mathbf{6 4} \oplus \mathbf{6 4}^{\prime}$ can be decomposed correspond to RR fluxes, whereas the other half are generalized NS fluxes. Of these, half are heterotic and half are ordinary fluxes, thus giving a very symmetric structure.

One can proceed analogously with the set of moduli $\mathbb{T}$. In this case they transform as a vectorial 7 of $S L(2, \mathbb{Z})^{7}$, as shown in table 7 . Let us define

$$
e^{i \mathbb{T}} \equiv 1+i \mathbb{T}-\mathbb{T} \otimes \mathbb{T}+\ldots
$$




\begin{tabular}{|c|c|c|c|}
\hline Flux parameter & Weight & Flux parameter & Weight \\
\hline $\bar{h}_{0}^{\prime}$ & $(-,-,-,-,-,-,-)$ & $e_{0}$ & $(+,+,+,+,+,+,+)$ \\
\hline$h_{0}$ & $(-,+,+,+,+,+,+)$ & $m^{\prime}$ & $(+,-,-,-,-,-,-)$ \\
\hline$-h_{i}$ & $(+, \overbrace{-,+,+},+,+,+)$ & $-\bar{f}_{i}^{\prime}$ & $(-, \overbrace{+,-,-},-,-,-)$ \\
\hline$e_{j}$ & $(+,+,+,+, \overbrace{i}^{-,+,+})$ & $\bar{a}_{j}^{\prime}$ & $(-,-,-,-, \overbrace{i}^{+,-,-})$ \\
\hline $\bar{h}_{i}^{\prime}$ & $(+, \overbrace{+,-,-}^{-},-,-,-)$ & $f_{i}$ & $(-, \overbrace{-,+,+}^{-},+,+,+)$ \\
\hline$q_{j}^{\prime}$ & $(\overbrace{+,-,-,}^{-})$ & $a_{j}$ & $\begin{array}{c}(-,+,+,+, \overbrace{i}^{-,+,+}) \\
j\end{array}$ \\
\hline $\bar{g}_{j i}^{\prime}$ & $(-, \overbrace{}^{+}$ & $b_{j i}$ & $\overbrace{}^{-,+,+}$ \\
\hline$a_{j}^{\prime}$ & $\begin{array}{c}(-,-,-,-, \overbrace{-,+,+}^{-\overbrace{}^{j}}) \\
\overbrace{}^{i} \overbrace{}^{j})\end{array}$ & $q_{j}$ & $\begin{array}{c}(+,+,+,+, \overbrace{+,-,-}^{+\overbrace{}^{j}}) \\
\overbrace{}^{i} \overbrace{}^{j})\end{array}$ \\
\hline$-g_{j i}$ & $(-, \overbrace{-,+,+}, \overbrace{-,+,+}^{\overbrace{j}})$ & $-\bar{b}_{j i}^{\prime}$ & $(+, \overbrace{+,-,-}, \overbrace{+,-,-}^{j})$ \\
\hline$-\bar{a}_{j}$ & $\left(\begin{array}{c}-,+,+,+, \overbrace{i,-,-}^{+-}) \\
j\end{array}\right.$ & $-e_{j}^{\prime}$ & $(+,-,-,-, \overbrace{i}^{-,+,+})$ \\
\hline$-\bar{b}_{j i}$ & $(+, \overbrace{-,+,+}, \overbrace{+,-,-}^{-})$ & $-g_{j i}^{\prime}$ & $\overbrace{+,-,-}, \overbrace{-,+,+}$ \\
\hline$-m$ & $\begin{array}{c}(+,+,+,+,-,-,-) \\
\underbrace{j}_{i}\end{array}$ & $-h_{0}^{\prime}$ & $\underbrace{(-,-,-,-,+,+,+)}_{i} \underbrace{j}$ \\
\hline$b_{j i}^{\prime}$ & $(+, \overbrace{\overbrace{i}^{+,-,}}^{\overbrace{i}}, \overbrace{-,+,+})$ & $\bar{g}_{j i}$ & $(-, \overbrace{-\overbrace{i}^{-,+,}, \overbrace{+,-,-}^{+}}^{\overbrace{}^{i}})$ \\
\hline$f_{i}^{\prime}$ & $(-, \overbrace{+,-,-},+,+,+)$ & $\bar{h}_{i}$ & $(+, \overbrace{-,+,+}^{-},-,-)$ \\
\hline$-e_{0}^{\prime}$ & $(+,-,-,-,+,+,+)$ & $-\bar{h}_{0}$ & $(-,+,+,+,-,-,-)$ \\
\hline$-\bar{f}_{i}$ & $(-, \overbrace{-,+,+}^{l},-,-,-)$ & $-h_{i}^{\prime}$ & $(+, \overbrace{+,-,-}^{\imath},+,+,+)$ \\
\hline
\end{tabular}

Table 6: Spinorial embedding of the background fluxes. The weights in each column correspond to one of the two Weyl spinors on which the set of fluxes $\mathbb{G}$ can be decomposed.

In this language, the superpotential (6.14) then takes the very compact form

$$
W=\left.\mathbb{G} \otimes e^{i \mathbb{T}}\right|_{(+,+,+,+,+,+,+)},
$$

which is reminiscent of the typical expressions for flux induced superpotentials. 


\begin{tabular}{c|c} 
Moduli & Weight \\
\hline \hline$S$ & $(1,0,0,0,0,0,0)$ \\
$T_{i}$ & $(0, \overbrace{1,0,0}^{i}, 0,0,0)$ \\
$U_{i}$ & $(0,0,0,0, \overbrace{1,0,0}^{i})$
\end{tabular}

Table 7: Embedding of the moduli in a 7 of $S L(2, \mathbb{Z})^{7}$.

Moreover, the Bianchi identities now correspond to constraints in the components of the bispinor of fluxes

$$
\mathbb{G} \otimes \mathbb{G}=\mathbb{G} \cdot \mathbb{G} \oplus \mathbb{G} \Gamma^{I_{1}} \mathbb{G} \oplus \ldots \oplus \mathbb{G} \Gamma^{I_{1} I_{2} I_{3} I_{4} I_{5} I_{6} I_{7}} \mathbb{G},
$$

where $\Gamma^{I_{1} \ldots I_{n}} \equiv \Gamma^{\left[I_{1}\right.} \ldots \Gamma^{\left.I_{n}\right]}$ and $\Gamma^{I}$ are the complexified gamma matrices of the relevant Clifford algebra, and $I_{a}=1, \overline{1}, \ldots, 7, \overline{7}$. 


\section{References}

[1] For a review and references see M. Graña Flux compactifications in string theory: A comprehensive review, Phys. Rept. 423 (2006) 91, hep-th/0509003.

[2] S. Gukov, C. Vafa and E. Witten, CFT's from Calabi-Yau Four-folds, Nucl. Phys. B584 (2000) 69, hep-th/9906070.

[3] S. Kachru and A. K. Kashani-Poor, Moduli potentials in type IIA compactifications with RR and NS flux, JHEP 0503 (2005) 066, hep-th/0411279.

[4] T. W. Grimm and J. Louis, The effective action of type IIA Calabi-Yau orientifolds, Nucl. Phys. B718 (2005) 153, hep-th/0412277.

[5] J. P. Derendinger, C. Kounnas, P. M. Petropoulos and F. Zwirner, Superpotentials in IIA compactifications with general fluxes, Nucl. Phys. B715 (2005) 211, hep-th/0411276; Fluxes and gaugings: $N=1$ effective superpotentials, hep-th/0503229.

[6] G. Villadoro and F. Zwirner, $N=1$ effective potential from dual type-IIA D6/O6 orientifolds with general fluxes, JHEP 0506 (2005) 047, hep-th/0503169.

[7] O. DeWolfe, A. Giryavets, S. Kachru and W. Taylor, Type IIA moduli stabilization, JHEP 0507 (2005) 066, hep-th/0505160.

[8] P. G. Cámara, A. Font and L. E. Ibáñez, Fluxes, moduli fixing and MSSM-like vacua in a simple IIA orientifold, JHEP 0509 (2005) 013, hep-th/0506066.

[9] P. G. Cámara, Fluxes, moduli fixing and MSSM-like vacua in type IIA string theory, hepth/0512239.

[10] C. M. Chen, T. Li and D. V. Nanopoulos, Type IIA Pati-Salam flux vacua, hep-th/0601064.

[11] J. Scherk and J. H. Schwarz, Spontaneous breaking of supersymmetry through dimensional reduction, Phys. Lett. B82 (1979) 60; How to get masses from extra dimensions, Nucl. Phys. B153 (1979) 61.

[12] N. Kaloper and R. C. Myers, The O(dd) story of massive supergravity, JHEP 9905 (1999) 010, hep-th/9901045. 
[13] G. Dall'Agata and S. Ferrara, Gauged supergravity algebras from twisted tori compactifications with fluxes, Nucl. Phys. B717 (2005) 223, hep-th/0502066.

[14] L. Andrianopoli, M. A. Lledó and M. Trigiante, The Scherk-Schwarz mechanism as a flux compactification with internal torsion, JHEP 0505 (2005) 051, hep-th/0502083.

[15] C. M. Hull and R. A. Reid-Edwards, Flux compactifications of string theory on twisted tori, hep-th/0503114.

[16] J. Shelton, W. Taylor and B. Wecht, Nongeometric flux compactifications, JHEP 0510 (2005) 085, hep-th/0508133.

[17] S. Hellerman, J. McGreevy and B. Williams, Geometric constructions of nongeometric string theories, JHEP 0401 (2004) 024, hep-th/0208174.

[18] A. Dabholkar and C. Hull, Duality twists, orbifolds, and fluxes, JHEP 0309 (2003) 054, hep-th/0210209.

[19] S. Kachru, M. B. Schulz, P. K. Tripathy and S. P. Trivedi, New supersymmetric string compactifications, JHEP 0303 (2003) 061, hep-th/0211182.

[20] C. M. Hull and A. Catal-Ozer, Compactifications with S-duality twists, JHEP 0310 (2003) 034, hep-th/0308133.

[21] A. Flournoy, B. Wecht and B. Williams, Constructing nongeometric vacua in string theory, Nucl. Phys. B706 (2005) 127, hep-th/0404217.

[22] M. B. Schulz, Superstring orientifolds with torsion: O5 orientifolds of torus fibrations and their massless spectra, Fortsch. Phys. 52 (2004) 963, hep-th/0406001.

[23] C. M. Hull, A geometry for non-geometric string backgrounds, JHEP 0510 (2005) 065, hep-th/0406102.

[24] A. Flournoy and B. Williams, Nongeometry, duality twists, and the worldsheet, JHEP 0601 (2006) 166, hep-th/0511126.

[25] A. Dabholkar and C. Hull, Generalised T-duality and non-geometric backgrounds, hepth/0512005. 
[26] A. Lawrence, M. B. Schulz and B. Wecht, D-branes in nongeometric backgrounds, hepth/0602025.

[27] P. Meessen and T. Ortin, An Sl(2,Z) multiplet of nine-dimensional type II supergravity theories, Nucl. Phys. B541 (1999) 195, hep-th/9806120.

[28] G. Dall'Agata, K. Lechner and M. Tonin, $D=10, N=I I B$ supergravity: Lorentz-invariant actions and duality, JHEP 9807 (1998) 017, hep-th/9806140.

[29] E. Eyras and Y. Lozano, Exotic branes and nonperturbative seven branes, Nucl. Phys. B573 (2000) 735 , hep-th/9908094.

[30] E. A. Bergshoeff, M. de Roo, S. F. Kerstan, T. Ortin and F. Riccioni, IIB nine-branes, hep-th/0601128.

[31] I. V. Lavrinenko, H. Lu and C. N. Pope, Fibre bundles and generalised dimensional reductions, Class. Quant. Grav. 15 (1998) 2239, hep-th/9710243.

[32] R. Blumenhagen, D. Lüst and T. R. Taylor, Moduli stabilization in chiral type IIB orientifold models with fluxes, Nucl. Phys. B663 (2003) 319, hep-th/0303016.

[33] J. F. G. Cascales and A. M. Uranga, Chiral $4 d N=1$ string vacua with D-branes and NSNS and RR fluxes, JHEP 0305 (2003) 011, hep-th/0303024; Chiral 4d string vacua with D-branes and moduli stabilization, hep-th/0311250.

[34] T. H. Buscher, A symmetry of the string background field equations, Phys. Lett. B194 (1987) 59.

[35] S. F. Hassan, T-duality, space-time spinors and $R$ - $R$ fields in curved backgrounds, Nucl. Phys. B568 (2000) 145, hep-th/9907152.

[36] S. Gurrieri, J. Louis, A. Micu and D. Waldram, Mirror symmetry in generalized Calabi-Yau compactifications, Nucl. Phys. B654 (2003) 61, hep-th/0211102.

[37] S. B. Giddings, S. Kachru and J. Polchinski, Hierarchies from fluxes in string compactifications, Phys. Rev. D66 (2002) 106006, hep-th/0105097.

[38] S. Kachru, M. B. Schulz and S. Trivedi, Moduli stabilization from fluxes in a simple IIB orientifold, JHEP 0310 (2003) 007, hep-th/0201028. 
[39] G. Dall'Agata and N. Prezas, Scherk-Schwarz reduction of M-theory on G2-manifolds with fluxes, JHEP 0510 (2005) 103, hep-th/0509052.

[40] D. D. Joyce, Compact Riemannian 7-manifolds with holonomy $G_{2}$. I, J. Differential Geometry (1996), No. 43 291-328; D. D. Joyce, Compact Riemannian 7-manifolds with holonomy $G_{2}$. II, J. Differential Geometry (1996), No. 43 329-375.

[41] C. Beasley and E. Witten, A note on fluxes and superpotentials in M-theory compactifications on manifolds of G(2) holonomy, JHEP 0207 (2002) 046, hep-th/0203061.

[42] T. House and A. Micu, M-theory compactifications on manifolds with G(2) structure, Class. Quant. Grav. 22 (2005) 1709, hep-th/0412006;

N. Lambert, Flux and Freund-Rubin superpotentials in M-theory, Phys. Rev. D71 (2005) 126001, hep-th/0502200;

A. Lukas and S. Morris, Moduli Kaehler potential for M-theory on a G(2) manifold, Phys. Rev. D69 (2004) 066003, hep-th/0305078.

[43] K. Becker, M. Becker, K. Dasgupta and P. S. Green, Compactifications of heterotic theory on non-Kaehler complex manifolds I, JHEP 0304 (2003) 007, hep-th/0301161.

[44] K. Becker, M. Becker, K. Dasgupta and S. Prokushkin, Properties of heterotic vacua from superpotentials, Nucl. Phys. B666 (2003) 144, hep-th/0304001.

[45] G. L. Cardoso, G. Curio, G. Dall'Agata and D. Lüst, BPS action and superpotential for heterotic string compactifications with fluxes, JHEP 0310 (2003) 004, hep-th/0306088; Heterotic string theory on non-Kaehler manifolds with H-flux and gaugino condensate, Fortsch. Phys. 52 (2004) 483, hep-th/0310021.

[46] S. Gurrieri, A. Lukas and A. Micu, Heterotic on half-flat, Phys. Rev. D70 (2004) 126009, hep-th/0408121.

[47] B. de Carlos, S. Gurrieri, A. Lukas and A. Micu, Moduli stabilisation in heterotic string compactifications, hep-th/0507173.

[48] S. Ferrara, D. Lüst, A. D. Shapere and S. Theisen, Modular invariance in supersymmetric field theories, Phys. Lett. B225 (1989) 363.

[49] L. Anguelova and K. Zoubos, Flux Superpotential in Heterotic M-theory, hep-th/0602039. 